\title{
Translationally invariant coupled cluster theory for simple finite systems
}

\author{
R. F. Bishop and M. F. Flynn \\ Department of Mathematics, University of Manchester Institute of Science and Technology, \\ P.O. Box 88, Manchester M60 1QD, United Kingdom \\ M. C. Boscá, E. Buendía, and R. Guardiola \\ Departamento de Física Moderna, Universidad de Granada, Facultad de Ciencias, 18071 Granada, Spain \\ (Received 9 February 1990)
}

\begin{abstract}
The widely used coupled cluster method (CCM) in quantum many-body theory has recently provided very accurate descriptions of a large number of extended systems. Although its earlier applications to closed-shell and neighboring finite nuclei were also very successful, they have been shrouded in algebraic and technical complexity. Furthermore, they are difficult to compare with more traditional calculations of generalized shell-model theory since, at least at the important level of two-body correlations, they have been largely implemented in relative-coordinate space rather than the more usual oscillator configuration space. The CCM is reviewed here in the precise context of applications to simple finite systems. Special attention is paid to formulate it in such a way that comparison may be made with generalized shell-model or configuration-interaction (CI) theories. Particular regard is paid to an exact incorporation of translational invariance, so that any spuriosity associated with the center-of-mass motion is always avoided. An important side benefit is that the number of many-body configurations in the usual oscillator basis is dramatically reduced. We are thereby able to present both $\mathrm{CI}$ and CCM calculations on ${ }^{4} \mathrm{He}$ up to the essentially unprecedented level of $60 \hbar \omega$ in oscillator excitation energy, for two popular and quasirealistic choices of the nucleon-nucleon interaction for which exact Monte Carlo results are available for this nucleus. Although even our simplest approximations attain about $95 \%$ of the total binding energy, the convergence in the oscillator configuration space is shown to be both very slow and of a complicated nonuniform nature. Strong implications are drawn for standard implementations of generalized shell-model techniques for heavier nuclei.
\end{abstract}

\section{INTRODUCTION}

The coupled cluster method (CCM) has become rather widely acknowledged over approximately the last decade as providing a widely applicable method for attacking the quantum many-body problem at a rather general and completely microscopic level. It has, by now, been very successfully applied to an extremely diverse array of physical systems, ranging from few-body, and larger, finite problems in nuclear physics ${ }^{1-10}$ and quantum chemistry, ${ }^{11-19}$ which nowadays include applications to relatively complex nuclei, atoms, and molecules, to various many-body (infinite) problems in both condensedmatter physics and (nonrelativistic and relativistic) quantum field theory. The former of these many-body applications includes such systems as the one-component Coulomb plasma (or electron gas), ${ }^{20-22}$ and nuclear matter, ${ }^{23,24}$ while the latter includes various anharmonic oscillators considered as $(0+1)$-dimensional model field theories, ${ }^{25-29}$ a model nucleon-pion field theory, ${ }^{30,31}$ and $\phi^{4}$-field theory in $(1+1)$ dimensions. ${ }^{32-36}$ In nearly all of these applications, the results obtained by the CCM are at least as accurate as those from any other microscopic formulation of the many-body problem, as pointed out in a recent review. ${ }^{37}$ In the majority of cases, the CCM provides results of the highest accuracy attained by fundamental methods.
The CCM formalism has also been further developed to deal with such topics as sum rules, ${ }^{38}$ pairing correlations and higher-order clustering in many-fermion systems, ${ }^{39,40}$ and a temperature-dependent formulation via the Bloch equation for the statistical density operator. ${ }^{41}$ Recently, a rather powerful extended version of the CCM has been formulated ${ }^{42,43}$ which has also permitted such applications as to the zero-temperature quantum hydrodynamics of a strongly interacting condensed Bose fluid, ${ }^{44}$ and to the problem of a charged impurity in a polarizable medium. ${ }^{45}$ This latter application is of particular relevance to the very important experimental tool of positron annihilation in metals, for example. The extended CCM has also been shown to be capable of describing the phases on both sides of a phase transition in an application $^{46}$ to the SU(2) quasispin model of Lipkin, Meshkov, and Glick. ${ }^{47}$ This model contains the spontaneous breakdown of parity as a good quantum number, and has been widely used to model the spherical to deformed shape transition in nuclei which occurs at high spins.

It is clear from the above discussion that while the $\mathrm{CCM}$ was originally invented by Coester and $\mathrm{Kümmel}^{48}$ within the framework of nuclear physics, its applications have nowadays extended very much further afield. Nevertheless, it seems both appropriate and timely to investigate anew some of the basic concepts of the method, particularly within the context of its applications to finite 
systems, and to discuss it in relation to the various configuration-interaction (CI) and other extended shellmodel calculations that have been very widely applied to nuclear systems after the older pioneering CCM work in nuclear physics. ${ }^{1-6}$ Since the underlying conceptual simplicity of the method has often been greatly obscured in much of the previous literature involving applications to nuclear physics, ${ }^{4-9}$ by the details of the technical machinery necessary to implement it, we concentrate in this paper on applications to the ${ }^{4} \mathrm{He}$ nucleus.

The main motivations for this work have been fourfold. Firstly, by concentrating on the four-body system and by considering only Wigner-type internucleon potentials, we can avoid the purely algebraic complications due to the discrete spin and isospin degrees of freedom and to antisymmetrization. Nevertheless, within these selfimposed constraints we shall present results for two widely used semirealistic microscopic potentials, namely the S3 potential of Afnan and Tang ${ }^{49}$ and the MT-V potential of Malfliet and Tjon. ${ }^{50}$ We stress from the outset that the restrictions imposed are by no means necessary in order to carry out CCM calculations. Their imposition does, however, considerably clarify the analysis of the main points that we wish to stress.

Our second, and perhaps most important, aim has been to show very explicitly and for the first time how the center-of-mass motion can be exactly removed within the various approximate implementations of the coupled cluster methodology considered here. We show that there is absolutely no spuriosity associated with the lack of either rotational or translational invariance in the formalism that we develop. Indeed, the exact incorporation of these invariances from the outset has the beneficial effect of greatly reducing the number of independent excitation amplitudes. Thirdly, by concentrating on the model ${ }^{4} \mathrm{He}$ problem, we shall be able to examine the convergence properties very critically as a function of the size of the harmonic-oscillator configuration space. Indeed, we shall present some results up to the almost unprecedented level of $60 \hbar \omega$ in oscillator excitation energy. Finally, by working with these simple four-body problems, we may also compare our results both with our own essentially exact results from the diffusion Monte Carlo method, ${ }^{51}$ and with those of other techniques. Of particular interest here is the comparison with more traditional extended shell-model calculations for this nucleus, ${ }^{52-55}$ which operate within a shell-model space restricted to considerably fewer quanta of excitation energy. Our results for the CCM convergence properties have wide-reaching implications for shell-model calculations in general.

The remainder of this paper is organized as follows. Section II presents a rather general overview of the CCM formalism. Particular attention is paid to its relationship with CI techniques and to how the formalism is both embedded within and yet transcends the general confines of either nondegenerate or degenerate perturbation theory. The formalism is specialized to the four-boson model problems considered here in Sec. III, and special emphasis is placed on the incorporation of translational (and rotational) invariance. The various calculational schemes that may be used to implement the method at different levels of approximation are described in Sec. IV, and the numerical results from them are presented in Sec. V. Finally, the results are compared with those of other calculations in Sec. VI, and their wider implications are critically discussed in Sec. VII.

\section{OVERVIEW OF COUPLED CLUSTER THEORY}

The coupled cluster method may be introduced within the context of many-body perturbation theory. For a given system this requires the assumption of an unperturbed basis (or model space) with a one-to-one correspondence with the particular physical states (or target space) being discussed. There is a wide choice for the model space, as has been discussed elsewhere. ${ }^{56} \mathrm{~A}$ prime consideration is the dimensionality $d$ of the model space, which must be the same as the number of eigenstates of the full Hamiltonian $H$ to be determined. In particular, in order to study the ground state of a closedshell atomic nucleus, we may choose $d$ to be unity.

A convenient formulation proceeds in terms of the effective interaction description. ${ }^{57}$ The goal is to determine a new Hamiltonian $H_{\text {eff }}$ which acts only in the model space and which generates the same set of $d$ eigenvalues as the full Hamiltonian $H$. Thus, in correspondence with the exact eigenvalue equation

$$
H\left|\Psi^{(n)}\right\rangle=E_{n}\left|\Psi^{(n)}\right\rangle, \quad n=1,2, \ldots, d,
$$

one seeks the equivalent equation

$$
H_{\mathrm{eff}}\left|\Psi_{0}^{(n)}\right\rangle=E_{n}\left|\Psi_{0}^{(n)}\right\rangle, \quad n=1,2, \ldots, d,
$$

where the states $\left|\Psi_{0}^{(n)}\right\rangle$ are the vectors in the model space. In this way one can write down an integral equation relating $H$ and $H_{\text {eff }}$, the so-called Bloch equation, ${ }^{58}$

$$
\Omega H_{\mathrm{eff}} P=H \Omega P,
$$

where $P$ is the projector onto the model space and $\Omega$ is the so-called wave operator which connects the model space with the physical space

$$
\Omega\left|\Psi_{0}^{(n)}\right\rangle=\left|\Psi^{(n)}\right\rangle, \quad n=1,2, \ldots, d .
$$

A useful generalized form of the Bloch equation has been given by Lindgren. ${ }^{12,59,60}$

Some properties of $\Omega$ are of special interest within the context of the CCM. Within the configurationinteraction method, ${ }^{61}$ or generalized shell-model theory, it is customary to introduce the correlation operator $F$ as

$$
\Omega=1+F,
$$

where $F$ is restricted to act only on ket states in the model space by the requirement $F=F P$. In nuclear physics applications, $F$ is thus conveniently parametrized in terms of linear combinations of several particle-hole excitations.

Without doubt the most important property of the wave operator is a consequence of the size extensivity ${ }^{13}$ of the energy of a many-body system. This requires the effective Hamiltonian to be additively separable over the 
possible subsystems in the various dissociation limits. In turn, this requires the wave operator to be multiplicatively separable. The importance of maintaining the separability conditions at any level of approximation has been stressed by Primas, ${ }^{62}$ although it was already realized by Brueckener $^{63}$ in the context of lowest-order RayleighSchrödinger perturbation theory.

Perhaps the most obvious way of ensuring the separability of $\Omega$ is to write it in the exponential form,

$$
\Omega=\exp (S),
$$

where the so-called cluster operator $S$ must be additively separable, and hence must correspond to a sum of connected diagrams. The long path which leads from perturbation theory to the exponential ansatz of Eq. (6), has its origins in the linked cluster theorem of Goldstone ${ }^{64}$ as well as in the analyses of perturbation series due to Hugenholtz ${ }^{65}$ and Hubbard ${ }^{66}$ in the case of closed-shell systems, and the corresponding linked valence theorem of Brandow $^{67}$ for open-shell systems. The $\exp (S)$ ansatz for the wave operator was first exploited by Coester and $\mathrm{Kümmel}^{48}$ within the context of closed-shell nuclear systems. It was later independently introduced into quantum chemistry by Čižek. ${ }^{11}$ Several extensions to openshell systems have been given by a number of authors, ${ }^{5,12,68}$ in the case of so-called complete model spaces, which contain all possible configurations of the valence particles over the valence orbitals.

A particularly important property of the $\exp (S)$ ansatz is that even when using an approximated (but connected) form for the operator $S$, the separability requirement is maintained. It is also clear why an approximate CI calculation with a correlation operator $F$ truncated at a certain level of excitations will fail to preserve the separability property of the exact wave function. Thus, after $F$ has been partitioned into its various $k$-body pieces $F_{k}$, $k=1,2, \ldots, A$ (with $A$ being the total number of particles), a typical CI calculation at the $\operatorname{SUB}(n)$ level will retain only those operators $\left\{F_{k}\right\}$ with $k \leq n$, and the higher-order partitions with $k>n$ are set to zero. On the other hand, the same $\operatorname{SUB}(n)$ truncation scheme, when performed instead on the cluster operator $S$, will still contain the excitation of multiple independent $k$-tuples which result from the higher powers of $S$ which arise from the expansion of the exponential. We remark finally that recently several workers have shown how the connectivity of the cluster operators and the effective Hamiltonian can even be maintained for open-shell systems in the case of incomplete model spaces. ${ }^{68-70}$

The violation of the separability property of the wave function and the consequent breakdown of size extensivity in $\operatorname{SUB}(n)$-truncated CI calculations that we have discussed above is only one particular example of the much wider phenomenon of approximate calculations not sharing the symmetries or other intrinsic properties of the exact system. A very well-known example of the breaking of symmetries is the Hartree-Fock solution of fermion systems, with its lack of translational invariance, and indeed even its violation of rotational invariance for deformed solutions. Similar problems can also occur in the CCM. ${ }^{10}$ The symmetry violations may originate here ei- ther with their violation by the model state or by their nonpreservation by the cluster operators $\left\{S_{k}\right\}$. A symmetry-conserving calculation must involve both a model space which satisfies the required symmetry and cluster operators which commute with the generators of the transformation. We note, however, that a complete (nontruncated) calculation, when performed either in the CCM or in the CI scheme, will restore any symmetry implicit in the Hamiltonian. On the other hand, such full (exact) calculations are seldom practicable.

The two symmetries of particular interest for present purposes are the invariances under spatial rotations and translations. Most calculations in nuclear physics have dealt with the problem of invariance under translations by using the internal Hamiltonian, $H \rightarrow H_{\text {int }}=H-T_{\text {c. m. }}$, where $T_{\text {c.m. }}$, is the kinetic-energy operator of the center of mass (c.m.), while continuing to use a model space which is not explicitly translationally invariant due to the complexity of using the appropriate set of intrinsic coordinates. This approach is essentially a compromise between solving the exact equations and neglecting the c.m. motion entirely. A similar problem also arises, in principle, with the angular-momentum operator $\mathbf{J}$ for Hamiltonians which are rotationally invariant. However, this problem often does not arise in practice since the singleparticle basis states are themselves usually chosen to be angular-momentum eigenstates. In this case there exist straightforward procedures to construct states of good total angular momentum.

All of the above points are specifically illustrated in the next section in the case of a four-boson system. In particular, the question of translational and rotational invariance is discussed in considerable detail.

\section{SPECIFIC FORMALISM FOR THE FOUR-BOSON MODEL PROBLEM}

As a concrete example of how the above general formalism may be implemented in practice, we now consider the lowest doubly closed-shell nucleus, namely, the fourbody ( $A=4$ ) problem of ${ }^{4} \mathrm{He}$. In order to keep the ensuing discussion as conceptually simple as possible, we also restrict ourselves from the outset to spin- and isospinindependent two-body forces, i.e., to pairwise interaction potentials of the Wigner type. The ${ }^{4} \mathrm{He}$ nucleus may thus be described in terms of a four-boson system in the sense that the spatial part of the wave function may be taken to be totally symmetric. The only role of the spin and isospin coordinates is hence to ensure overall antisymmetry. For all other purposes they may essentially be ignored.

In view of the closed-shell nature of the $A=4$ nuclear system and the fact that we shall constrain the discussion to the ground state only, we therefore henceforth specialize to the simplest case where the model space is one dimensional. The projection operator is thus given as $P=|\Phi\rangle\langle\Phi|$ in terms of the single model-space or uncorrelated four-body spatial wave function $|\Phi\rangle$. In order to construct $|\Phi\rangle$ and all other operators, we need first to choose a suitable single-particle basis. Our choice is the standard orthonormal harmonic-oscillator basis

$\{|n l m\rangle ; n=0,1,2, \ldots ; l=0,1, \ldots, n ;-l \leq m \leq l\}$, 
with a free parameter $\alpha=(m \omega / \hbar)^{1 / 2}$. In terms of the vacuum state $|0\rangle$, we may write these single-particle wave functions in the form

$$
|n l m\rangle=a_{n l m}^{\dagger}|0\rangle
$$

where the creation operators $\left\{a_{n l m}^{\dagger}\right\}$ and their Hermitian conjugates (the destruction operators) obey the usual bosonic canonical commutation relations

$$
\begin{aligned}
& {\left[a_{n l m}, a_{n^{\prime} l^{\prime} m^{\prime}}^{\dagger}\right]=\delta_{n n^{\prime}} \delta_{l l^{\prime}} \delta_{m m^{\prime}},} \\
& {\left[a_{n l m}^{\dagger}, a_{n^{\prime} l^{\prime} m^{\prime}}^{\dagger}\right]=0 .}
\end{aligned}
$$

The r-space representation of these single-particle wave functions is given as usual in terms of the spherical harmonics and the associated Laguerre polynomials, $L_{n}^{l+1 / 2}\left(\alpha^{2} r^{2}\right) .^{71}$

The obvious choice for the $A=4$ model wave function $|\Phi\rangle$ is thus where each of the four uncorrelated nucleons is put into the $0 s$ orbital,

$$
\begin{aligned}
& |\Phi\rangle=(4 !)^{-1 / 2}\left(a_{000}^{\dagger}\right)^{4}|0\rangle, \\
& \langle\Phi \mid \Phi\rangle=1 .
\end{aligned}
$$

Its $\mathbf{r}$-space representation is most easily given in terms of the field operators

$$
\widehat{\psi}(\mathbf{r}) \equiv \sum_{\beta} \psi_{\beta}(\mathbf{r}) a_{\beta}
$$

where the single index $\beta$ is used henceforth to represent the set of indices $(\mathrm{nlm})$. The symmetrized four-particle position bra vector may be written as

$$
\left\langle\mathbf{r}_{1} \mathbf{r}_{2} \mathbf{r}_{3} \mathbf{r}_{4}\right| \equiv(4 !)^{-1 / 2} \prod_{i=1}^{4}\langle 0| \hat{\psi}\left(\mathbf{r}_{i}\right) \text {. }
$$

We thus have the explicit form

$$
\begin{aligned}
\left\langle\mathbf{r}_{1} \mathbf{r}_{2} \mathbf{r}_{3} \mathbf{r}_{4} \mid \Phi\right\rangle & =\prod_{i=1}^{4} \psi_{000}\left(\mathbf{r}_{i}\right) \\
& =\frac{\alpha^{6}}{\pi^{3}} \exp \left(-\frac{1}{2} \alpha^{2} \sum_{i=1}^{4} r_{i}^{2}\right) .
\end{aligned}
$$

If we define the center-of-mass position coordinate of the $A$-body system in the usual way as

$$
\mathbf{R} \equiv \frac{1}{A} \sum_{i=1}^{A} \mathbf{r}_{i}
$$

Eq. (12) may be trivially reexpressed in either of the equivalent forms,

$$
\begin{aligned}
\left\langle\mathbf{r}_{1} \mathbf{r}_{2} \mathbf{r}_{3} \mathbf{r}_{4} \mid \Phi\right\rangle= & \frac{\alpha^{6}}{\pi^{3}} \exp \left[-\frac{1}{2} \alpha^{2} \sum_{i=1}^{4}\left(\mathbf{r}_{i}-\mathbf{R}\right)^{2}\right] \\
& \times \exp \left(-\frac{1}{2} \alpha^{2} A R^{2}\right) \\
= & \frac{\alpha^{6}}{\pi^{3}} \exp \left[-\frac{\alpha^{2}}{2 A} \sum_{i<j=1}^{4} r_{i j}^{2}\right] \\
& \times \exp \left(-\frac{1}{2} \alpha^{2} A R^{2}\right)
\end{aligned}
$$

The uncorrelated wave function is thus the simple product of an intrinsic wave function with a 0 s harmonic-oscillator wave function for the c.m. motion. The intrinsic wave function is readily seen from the explicit forms of Eq. (14) to be both translationally invariant and rotationally invariant. Our immediate goal is now to construct a correlated wave function within the CCM which can be decomposed in an analogous way and without exciting the c.m. motion. In this way, the c.m. motion can be removed absolutely unambiguously and the remaining intrinsic wave function can be guaranteed to be invariant under rotations and translations.

Turning firstly, therefore, to the construction of the cluster operator $S$ for the $\boldsymbol{A}$-body problem, we immediately partition it into its various $k$-body pieces as

$$
S=\sum_{k=1}^{A} S^{(k)}
$$

Furthermore, from our prior discussion, we need only consider operators $S^{(k)}$ such that, in our secondquantized notation,

$S^{(k)}=\sum_{\beta_{1}}^{\prime} \cdots \sum_{\beta_{k}}^{\prime} S^{(k)}\left(\beta_{1}, \ldots, \beta_{k}\right) a_{\beta_{1}}^{\dagger} \cdots a_{\beta_{k}}^{\dagger} a_{000}^{k}$.

The primes on the sums in Eq. (16) over the singleparticle labels remind us that the indices $\left\{\beta_{i}\right\} \equiv\left\{\left(n_{i} l_{i} m_{i}\right)\right\}$ run over all "unoccupied" orbitals only, i.e., the term $\beta_{i}=(0,0,0)$ is excluded for each value of $i$.

For most practical calculations within the CCM, the resulting set of (nonlinear) equations must be truncated. We have described one such truncation scheme, namely, the $\operatorname{SUB}(n)$ scheme in which all operators $S^{(k)}$ with $k>n$ are set to zero, and the remaining approximate equations for the set of matrix elements $\left\{S^{(k)}\left(\beta_{1}, \ldots, \beta_{k}\right) ; k=1,2, \ldots, n\right\}$ are solved exactly. In our ensuing discussion we shall assume the $\operatorname{SUB}(2)$ approximation, in which, for the $A=4$ problem, the proper (connected) three- and four-body correlations are neglected. Since it will transpire that in order to preserve translational and rotational symmetry, the matrix elements of the one-body and two-body partitions $S^{(1)}$ and $S^{(2)}$ are strongly coupled, it will be convenient to write our SUB(2)-approximated cluster operator from the outset as

$$
S \rightarrow S^{(1,2)}=\sum_{\beta_{1}} \sum_{\beta_{2}} S\left(\beta_{1} \beta_{2}\right) a_{\beta_{1}}^{\dagger} a_{\beta_{2}}^{\dagger} a_{000}^{2},
$$

where the primes on the summations have been removed to indicate that the double sum may now include terms where either (but not both) $\beta_{1}$ or $\beta_{2}$ may be the "occupied" (or hole-state) index $(\mathrm{nlm}) \rightarrow(000)$. The terms $S\left(\beta_{1} \beta_{2}\right)$ with $\beta_{1}$ and $\beta_{2}$ both unoccupied (or particle-state) labels then refer to the real two-body correlations, whereas those with either $\beta_{1}$ or $\beta_{2}$ the occupied (000) label refer to the one-body correlations. We also remind the reader that the $S^{(1)}$ partition of the cluster operator may be regarded in the light of the well-known Thouless theorem ${ }^{72}$ as being responsible for an arbitrary change of the single-particle basis. Alternatively, an unrestricted SUB(1) approximation leads to the usual Hartree approx- 
imation in the present bosonic case.

Let us now consider the state $S^{(1,2)}|\Phi\rangle$. It is well known that an arbitrary admixture of (1p-1h and) $2 \mathrm{p}-2 \mathrm{~h}$ states is not, in general, either rotationally invariant or translationally invariant. Rotational invariance may rather easily be imposed, however, by coupling the angu- lar momenta of the two excited particles to states $|\lambda \mu\rangle$ of good total angular moment $\lambda=0$ and its projection $\mu=0$ onto a fixed axis, in terms of the usual Clebsch-Gordan coefficients. Thus, after the imposition of rotational invariance, our cluster operator has fewer independent matrix elements,

$$
\begin{aligned}
S^{(1,2)} & \rightarrow \sum_{n_{1} n_{2} l_{1} m_{1}} S\left(n_{1} l_{1} m_{1}, n_{2} l_{1}-m_{1}\right)\left\langle l_{1} m_{1} l_{1}-m_{1} \mid l_{1} l_{1} 00\right\rangle\left[a_{n_{1} l_{1}}^{\dagger} \times a_{n_{2} l_{1}}^{\dagger}\right]_{0}^{0} a_{000}^{2} \\
& \equiv \sum_{n_{1} n_{2} l_{1}} S\left(n_{1} n_{2} l_{1}\right)\left[a_{n_{1} l_{1}}^{\dagger} \times a_{n_{2} l_{1}}^{\dagger}\right]_{0}^{0} a_{000}^{2} .
\end{aligned}
$$

Translational invariance may now also be imposed by recoupling the product of single-particle harmonic-oscillator states into sums of comparable products of oscillator states for the relative and c.m. motion of the pair. This is accomplished as usual in terms of the well-known Brody-Moshinsky brackets, ${ }^{73}$ defined for operators acting on the vacuum state as

$$
\left[a_{n_{1} l_{1}}^{\dagger} \times a_{n_{2} l_{2}}^{\dagger}\right]_{\mu}^{\lambda}|0\rangle=\sum_{n l N L}\left\langle n l N L \lambda \mid n_{1} l_{1} n_{2} l_{2} \lambda\right\rangle\left[a_{\mathrm{rel} ; n l}^{\dagger} \times a_{\mathrm{c} . \mathrm{m} . ; N L}^{\dagger}\right]_{\mu}^{\lambda}|0\rangle
$$

where the indices $(n l)$ and $(N L)$ refer, respectively, to the relative and c.m. motion of the pair, as indicated. Equation (18) may thus be rewritten as

$$
S^{(1,2)}=\sum_{n_{1} n_{2} l_{1}} S\left(n_{1} n_{2} l_{1}\right) \sum_{n l N L}\left\langle n l N L 0 \mid n_{1} l_{1} n_{2} l_{1} 0\right\rangle\left[a_{\mathrm{rel} ; n l}^{\dagger} \times a_{\mathrm{c} . \mathrm{m} . ; N L}^{\dagger}\right]_{0}^{0} a_{000}^{2} .
$$

This operator will only produce a translationally invariant state $S^{(1,2)}|\Phi\rangle$ if the c.m. motion of the pair destroyed by the operator $a_{000}^{2}$ acting on $|\Phi\rangle$ (i.e., the two holes) is the same as that of the pair of particles created by the product of the two creation operators for each term in the sum. The product $a_{000}^{2}$ can clearly itself be reexpressed in terms of a product of operators for the relative and c.m. motion. It is clear that both of these latter operators will destroy a $0 s$ harmonic-oscillator state in the relevant variable. Thus, translational invariance is guaranteed if and only if the sums in Eq. (20) are restricted to terms with $N=L=0$. The angular momentum coupling then further requires that $l=0$ also. In this way we are led to the cluster operator

$$
\begin{aligned}
S^{(1,2)} & \rightarrow \sum_{n} \sum_{n_{1} n_{2} l_{1}} S\left(n_{1} n_{2} l_{1}\right)\left\langle n 0000 \mid n_{1} l_{1} n_{2} l_{1} 0\right\rangle\left[a_{\mathrm{rel} ; n 0}^{\dagger} \times a_{\mathrm{c} . \mathrm{m} . ; 00}^{\dagger}\right]_{0}^{0} a_{000}^{2} \\
& \equiv \sum_{n} S_{n}\left[a_{\mathrm{rel} ; n 0}^{\dagger} \times a_{\mathrm{c} . \mathrm{m} . ; 00}^{\dagger}\right]_{0}^{0} a_{000}^{2} \\
& \equiv \sum_{n=1}^{\infty} S_{n} \sum_{n_{1} n_{2} l_{1}}\left\langle n 0000 \mid n_{1} l_{1} n_{2} l_{1} 0\right\rangle\left[a_{n_{1} l_{1}}^{\dagger} \times a_{n_{2} l_{1}}^{\dagger}\right]_{0}^{0} a_{000}^{2},
\end{aligned}
$$

where, in the last form, we have used the reality and orthonormality of the Brody-Moshinsky coefficients.

We note firstly that the imposition of the invariance with respect to spatial translations and rotations has drastically reduced the number of independent matrix elements. Secondly, we note that the terms in Eq. $(21)$ with $\left(n_{1}, l_{1}\right)=(0,0)$ or $\left(n_{2}, l_{1}\right)=(0,0)$ must clearly be included for the invariances to hold. These terms give precisely the $1 \mathrm{p}-1 \mathrm{~h}$ excitations as explained above. Finally, the sum does not include the term with both $\left(n_{1}, l_{1}\right)=(0,0)$ and $\left(n_{2}, l_{1}\right)=(0,0)$, since this can only occur for $n=0$ also, and this case is excluded as it simply reproduces the uncorrelated state $|\Phi\rangle$.

The explicit coordinate-space representation of the wave function generated by the cluster operator of Eq. (21) may also be rather easily found. One must project over the four-particle position bra vector of Eq. (11), and after some standard transformations we easily obtain the final result,

$$
\left\langle\mathbf{r}_{1} \mathbf{r}_{2} \mathbf{r}_{3} \mathbf{r}_{4}\left|S^{(1,2)}\right| \Phi\right\rangle=\frac{\alpha^{6}}{\pi^{3}} \exp \left[-\frac{1}{2} \alpha^{2} \sum_{k=1}^{4} r_{k}^{2}\right) 2 \sum_{n=1}^{\infty} S_{n} \sum_{i<j=1}^{4}\left[\frac{2^{n} n !}{(2 n+1) ! !}\right]^{1 / 2} L_{n}^{1 / 2}\left(\frac{1}{2} \alpha^{2} r_{i j}^{2}\right) .
$$

By comaparison with Eqs. (12) and (14), we observe that the c.m. motion factors out exactly as in the uncorrelated wave function, and the remaining intrinsic wave function is manifestly invariant under translations and rotations as now expected. An equivalent representation to Eq. (22) is the somewhat simpler form

$$
\begin{aligned}
& \left\langle\mathbf{r}_{1} \mathbf{r}_{2} \mathbf{r}_{3} \mathbf{r}_{4}\left|S^{(1,2)}\right| \Phi\right\rangle \\
& =\sum_{n=0}^{\infty} C_{n} \sum_{i<j=1}^{4}\left(r_{i j}\right)^{2 n} \exp \left(-\frac{1}{2} \alpha^{2} \sum_{k=1}^{4} r_{k}^{2}\right),
\end{aligned}
$$

where the coefficients $\left\{C_{n}\right\}$ may easily be constructed in 
terms of the corresponding parameters $\left\{S_{n}\right\}$ by making use of the explicit form of the associated Laguerre polynomials.

We note, in particular, that the step leading to Eq. (22) may be performed because we are indeed summing over all values of the indices $n_{1}, n_{2}$, and $l_{1}$ which lead to a nonzero value for the Brody-Moshinsky bracket $\left\langle n 0000 \mid n_{1} l_{1} n_{2} l_{1} 0\right\rangle$, i.e., over all values which satisfy the (number of quanta) relation,

$$
n_{1}+n_{2}+l_{1}=n \text {. }
$$

Most importantly, the specific terms with $n_{1}=l_{1}=0$, $n_{2}=n$, and $n_{2}=l_{1}=0, n_{1}=n$ must be included. It is precisely these terms which correspond to the effective $1 \mathrm{p}-1 \mathrm{~h}$ excitations, i.e., to the partition $S^{(1)}$ of the cluster operator $S$. The presence of these terms also leads to one final minor complication when the quadratic term $\frac{1}{2}\left(S^{(1,2)}\right)^{2}$ arising from the $\exp (S)$ form of the CCM wave function is now also considered. Thus, simply because the linear term in $S^{(1,2)}$ leads to a state which is invariant under rotations and translations, we are by no means guaranteed that so does the corresponding quadratic term. When carrying out the projection over the four-particle position bra vector for the quadratic term $\frac{1}{2}\left(S^{(1,2)}\right)^{2}$, there are two distinct classes of contractions. In the first place are those terms in which none of the creation operators in the representation of Eq. (21) for either $S^{(1,2)}$ operator is contracted with an (occupied-state) destruction operator, even if its own index takes the occupied-state value (000). The set of such contractions is readily seen to lead to a wave function analogous to Eq. (22), but now with a product of two Laguerre polynomials, one involving the relative coordinate $\mathbf{r}_{i j}$ as before, and the other the relative coordinate $\mathbf{r}_{k l}$ of the remaining pair once particles $i$ and $j$ have been selected. Such terms again clearly lead to a wave function which is invariant under rotations and translations.

There remains, however, a group of terms which correspond to the case where at least one of the creation operators in the representation of Eq. (21) of either of the two $S^{(1,2)}$ terms bears the index $(000)$ and is contracted with one of the corresponding destruction operators. It is simple to show that such terms correspond to an excitation of the c.m. motion, and hence to a wave function which is not invariant under translations. Nevertheless, such unwanted terms are easily excluded by the simple device of taking the $\exp \left(S^{(1,2)}\right)$ representation of the wave operator $\Omega$ in normal-ordered form. Furthermore, once this has been done, the cubic (and higher-order) terms in the expansion of the exponential now become identically zero for the $A=4$ system when acting on the model state $|\Phi\rangle$, thereby restoring the original property of the usual formulation of the CCM for this system. Such a normal ordering leaves all of the usual characteristics and features of the CCM quite unchanged, and indeed also arises quite naturally within the open-shell CCM formulation. $^{12}$

Thus, we may summarize our discussion to date. The most general CCM wave function at the SUB(2) level of approximation for the $A=4$ bosonic system under consideration, which is invariant under rotations and translations, has been found to be given by

$$
\left|\Psi_{2}\right\rangle=\exp \left(S^{(1,2)}\right):|\Phi\rangle,
$$

with a cluster operator $S^{(1,2)}$ given by Eq. (21). It also has the more explicit form

$$
\begin{aligned}
\left|\Psi_{2}\right\rangle=(4 !)^{-1 / 2}[ & \left(a_{000}^{\dagger}\right)^{4}+12 \sum_{n=1}^{\infty} S_{n} \theta_{n} \\
& \left.+12 \sum_{n=1}^{\infty} \sum_{n^{\prime}=1}^{\infty} S_{n} S_{n^{\prime}} \theta_{n n^{\prime}}\right]|0\rangle,
\end{aligned}
$$

where the operators $\theta_{n}$ and $\theta_{n n^{\prime}}$ are defined as

$$
\begin{aligned}
\theta_{n} \equiv \sum_{n_{1} n_{2} l}\left\langle n 0000 \mid n_{1} l n_{2} l 0\right\rangle\left[a_{n_{1} l}^{\dagger} \times a_{n_{2} l}^{\dagger}\right]_{0}^{0}\left(a_{000}^{\dagger}\right)^{2}, \\
\theta_{n n^{\prime}} \equiv \sum_{n_{1} n_{2} l} \sum_{n_{1^{\prime} n_{2}^{\prime} l^{\prime}}}\left\langle n 0000 \mid n_{1} l n_{2} l 0\right\rangle \\
\times\left\langle n^{\prime} 0000 \mid n_{1}^{\prime} l^{\prime} n_{2}^{\prime} l^{\prime} 0\right\rangle \\
\times\left[a_{n_{1} l}^{\dagger} \times a_{n_{2} l}^{\dagger}\right]_{0}^{0}\left[a_{n_{1}^{\prime} l^{\prime}}^{\dagger} \times a_{n_{2}^{\prime} l^{\prime}}^{\dagger}\right]_{0}^{0},
\end{aligned}
$$

and where the normal ordering has explicitly been taken into account.

The coordinate-space representation of the wave function given by Eq. (26) is

$$
\left\langle\mathbf{r}_{1} \mathbf{r}_{2} \mathbf{r}_{3} \mathbf{r}_{4} \mid \Psi_{2}\right\rangle=\frac{\alpha^{6}}{\pi^{3}} \exp \left[-\frac{1}{2} \alpha^{2} \sum_{m=1}^{4} r_{m}^{2}\right]\left[1+2 \sum_{n=1}^{\infty} S_{n} \sum_{i<j=1}^{4} \mathcal{F}_{n}\left(r_{i j}\right)+2 \sum_{n=1}^{\infty} \sum_{n^{\prime}=1}^{\infty} S_{n} S_{n^{\prime}} \sum_{i<j=1}^{4} \mathcal{F}_{n}\left(r_{i j}\right) \mathcal{F}_{n^{\prime}}\left(r_{k l}\right)\right],
$$

where

$$
\mathcal{F}_{n}(r) \equiv\left[\frac{2^{n} n !}{(2 n+1) ! !}\right]^{1 / 2} L_{n}^{1 / 2}\left(\frac{1}{2} \alpha^{2} r^{2}\right)
$$

and where, in the last term in Eq. (28), the notation for the unsummed indices is that the pair $\{k l\}$ represents the two remaining particles after the pair $\{i j\}$ has been chosen. The wave function of Eq. (28) may finally also be written in the equivalent form

$$
\begin{aligned}
\left\langle\mathbf{r}_{1} \mathbf{r}_{2} \mathbf{r}_{3} \mathbf{r}_{4} \mid \Psi_{2}\right\rangle= & \frac{\alpha^{6}}{\pi^{3}}\left[1+\sum_{i<j=1}^{4} h\left(r_{i j}\right)\right. \\
& \left.+\frac{1}{2} \sum_{i<j=1}^{4} h\left(r_{i j}\right) h\left(r_{k l}\right)\right] \\
& \times \exp \left[-\frac{1}{2} \alpha^{2} \sum_{m=1}^{4} r_{m}^{2}\right]
\end{aligned}
$$


in terms of the two-body cluster correlation function $h(r)$ defined as

$$
h(r) \equiv 2 \sum_{n=1}^{\infty} S_{n} \mathcal{F}_{n}(r)
$$

Before proceeding to describe our numerical calculations, we first make some observations on both the above SUB(2)-approximated CCM formalism and its simpler linearized version in which the terms quadratic in the coefficients $\left\{S_{n}\right\}$ or, equivalently, in the correlation function $h(r)$, are neglected. This linearized subapproximation is clearly equivalent to the corresponding CI treatment at the SUB(2) level implementation.

For either the CCM or the CI method to be utilized in practice at this SUB(2) level, we thus have to solve either a coupled set of algebraic equations for the coefficients $\left\{S_{n}\right\}$ from the representation in either Eq. (26) or Eq. (28), or some suitable integro-differential equation for the function $h(r)$ in Eq. (30). In the CCM these equations are intrinsically nonlinear, whereas in the corresponding CI approximations the analogous equations are linear. Since it is one of our primary aims in the present work to compare the present CCM techniques with those of other generalized shell-model approaches, we shall be mainly interested here in working directly in the harmonicoscillator basis by using either Eq. (26) or Eq. (28) as our starting point. The former Fock-space form is particularly suited to the standard shell-model machinery as described in Sec. IV and Appendix A. The explicit coordinate-space representation of Eq. (28) is also useful in cases where one may readily utilize various properties of the associated Laguerre polynomials and their generating function. Further details of this approach, which is especially useful in the case of two-body potentials which are specified as a linear combination of Gaussian factors, are given in Appendix B.

It is clear that whenever the CCM representations of Eqs. (26) or (28) or their linearized CI counterparts are utilized in practice, the otherwise infinite sums over the principal oscillator quantum number must be truncated at some maximal value $n_{\max }$, in common with all other comparable generalized shell-model schemes. We first note that such a SUB(2) calculation in either the CI or CCM scheme is equivalent to considering $2 \mathrm{p}-2 \mathrm{~h}$ configurations up to a total excitation energy of $2 n_{\max } \hbar \omega$ (with $\omega \equiv \hbar \alpha^{2} / m$ ). The imposition of translational and rotational invariance has dramatically reduced the number of corresponding CI basis states to just $n_{\max }+1$, which is far fewer than in most conventional CI calculations. For example, in a conventional full CI calculation of ${ }^{4} \mathrm{He}^{54}$ the number of fully antisymmetric positiveparity basis states, all of which are constructed so as to have good total angular momentum and isospin, is equal to $7,45,221,853$, and 2765 , respectively, in the $2 n_{\max } \hbar \omega$ spaces with $n_{\max }=1,2,3,4$, and 5 . This basis size grows so rapidly with $n_{\max }$ that most earlier calculations have been restricted to much smaller values of $n_{\max }$ than are now considered in the detailed calculations reported below.

Secondly, in contrast with what seems to be a widely held opinion to the contrary, and which stems from a misunderstanding of the true situation, ${ }^{74}$ it is not necessary to work with a basis which is complete in the sense that it includes all possible states of all possible configurations up to a given amount $N \hbar \omega$ of oscillator excitation energy in order to avoid spuriosity in connection with the c.m. motion. Thus, we have explicitly demonstrated that a selected set of $2 \mathrm{p}-2 \mathrm{~h}$ configurations with a prescribed $1 \mathrm{p}-1 \mathrm{~h}$ admixture can provide an incomplete space in which the c.m. motion may also be exactly removed. Finally, our findings correspond to a very simplified version of the group-theoretical methods ${ }^{75}$ which have been designed to select many-particle configurations in the harmonic-oscillator basis which preserve translational invariance.

As a last point, we note that the discussion above has focused on the expansion of the CI or CCM equations in terms of the oscillator basis from which it originated. This is in keeping with one of our main aims which is to maintain as close contact as possible with other generalized shell-model schemes, which also employ a truncated oscillator basis. Nevertheless, as can be seen from Eq. (30), the formulation can also be completely given in terms of the coordinate-space representation, where the cluster operator $S$ is now represented by the cluster correlation function $h(r)$ in the coordinate basis rather than in terms of the cluster amplitudes $\left\{S_{n}\right\}$ in the oscillator basis. It is purely a matter of calculational convenience to decide which representation to use. In practice, one needs to truncate both spaces, either by some choice of $n_{\max }$ in the oscillator basis or by some analogous device in $r$ space. The rate of convergence of the numerical scheme with respect to this truncation parameter is what then usually leads us to prefer one representation over another. One of our main conclusions from the numerical results presented below is that the convergence in the (usual shell-model) oscillator basis is inordinately slow in comparison with equivalent calculations in the coordinate-space basis.

\section{THE CALCULATIONAL SCHEMES}

The CCM parametrization of Eq. (26) may now be used in various different ways in order to perform several distinct approximate calculations of the ground-state energy eigenvalue $E$ of the bosonic $A=4$ system. It is of considerable interest to compare and contrast these various approximations, which we therefore now describe.

The simplest calculation is that of the corresponding CI method at the same SUB(2) level of approximation in which the last term of Eq. (26) is neglected and only the remaining $(1 \mathrm{p}-1 \mathrm{~h}$ plus) $2 \mathrm{p}-2 \mathrm{~h}$ configurations up to $2 n_{\max } \hbar \omega$ in oscillator excitation energy are considered. The equivalent coordinate-space representation is given by Eqs. (12) and (22). The (approximate) CI eigenvalue equation is thus

$$
(H-E)\left[\left(a_{000}^{\dagger}\right)^{4}+12 \sum_{n=1}^{n_{\max }} S_{n} \theta_{n}\right]|0\rangle=0 .
$$

By projection of Eq. (32) onto the uncorrelated ground 
state $\left(a_{000}^{\dagger}\right)^{4}|0\rangle$ and onto all independent and invariant (1p-1h and) $2 \mathrm{p}-2 \mathrm{~h}$ states $\theta_{n}|0\rangle$ with $n \leq n_{\max }$, we have a system of $n_{\max }+1$ linear equations to be solved for the unknown coefficients $\left\{S_{n} ; n=1,2, \ldots, n_{\max }\right\}$ and the energy eigenvalue $E$. This set of equations is fully equivalent to its usual formulation of diagonalizing the Hamiltonian in the truncated (and unnormalized) basis. For future reference we denote the above method as the CI2 method. The resulting approximation for the energy eigenvalue from such a CI calculation is a strict upper bound to the exact value, and the estimate is a monotonically decreasing function of the configuration-space size parameter $n_{\max }$. The particular interest in this kind of calculation is that we may fairly readily go to very high values of $n_{\max }$ in order to examine the rate of convergence as a function of the size of the configuration space.

The standard CCM equation at this SUB(2) level of approximation is similarly obtained by projecting the approximate Schrödinger equation $(H-E)\left|\Psi_{2}\right\rangle=0$, where $\left|\Psi_{2}\right\rangle$ is given by the full Eq. (26), onto the set of states $|\Phi\rangle$ and $\theta_{n}|0\rangle$ with $n \leq n_{\max }$. The resulting set of $n_{\max }+1$ approximate equations is now nonlinear in the amplitudes $\left\{S_{n}\right\}$ and linear in the energy eigenvalue $E$. The resulting CCM estimate for $E$ will not, in general, provide an upper bound to its exact counterpart, unlike the corresponding above $\mathrm{CI}$ calculation which is its linearized version. A variant of this standard CCM approach consists in calculating the expectation value of the Hamiltonian $\left\langle\Psi_{2}|H| \Psi_{2}\right\rangle /\left\langle\Psi_{2} \mid \Psi_{2}\right\rangle$ in the state $\left|\Psi_{2}\right\rangle$ of Eq. (26) and with the coefficients $\left\{S_{n}\right\}$ calculated in the standard CCM scheme above. A comparison of these two CCM estimates, where the latter is now a strict upper bound, thereby also provides an internal consistency check on the accuracy of the CCM. We shall henceforth refer to these two CCM computational procedures by the respective abbreviations $\mathrm{CC} 2$ and $\mathrm{CC} 2-\mathrm{E}$.

We may also envisage extending the above CCM scheme in SUB(2) approximation to an extended variational method by replacing the products $\left\{S_{n} S_{n^{\prime}}\right\}$ in Eq. (26) with a new set of independent amplitudes $\left\{S_{n n^{\prime}}\right\}$, and then minimizing the expectation value of the Hamiltonian in this basis. This extended variational calculation is clearly also completely equivalent to an extended CI calculation in the correspondingly enlarged (but still incomplete) basis that now also includes (some) $4 \mathrm{p}-4 \mathrm{~h}$ excitations, namely, those that comprise two independent $2 \mathrm{p}-2 \mathrm{~h}$ excitations. It will henceforth be referred to as the restricted CI4 method, or by the abbreviation CI4-R. Finally, a restricted version of the above procedure would be to minimize the expectation value $\left\langle\Psi_{2}|H| \Psi_{2}\right\rangle /\left\langle\Psi_{2} \mid \Psi_{2}\right\rangle$ directly in the basis of Eq. (26) with respect to the parameters $\left\{S_{n}\right\}$. We denote this variational procedure by the abbreviation CC2-V. A comparison of this estimate with its standard CCM counterpart again provides an internal check on the accuracy of the approximation,

We remind the reader that in each of the above calculational schemes, apart from the number $n_{\max }$ of states in the basis, the harmonic-oscillator parameter $\alpha$ is also still a free parameter. In those calculations which have a variational basis, the optimal choice for $\alpha$ is clearly that which minimizes the energy estimate. One of the particularly interesting outcomes of the numerical results presented in Sec. $\mathrm{V}$ is their dependence on the oscillator parameter, as discussed below in more detail.

\section{RESULTS}

We now present the numerical results for the groundstate energy of the ${ }^{4} \mathrm{He}$ nucleus corresponding to two popular choices of the nucleon-nucleon potential which contain only Wigner components. These are the Wigner part of the S3 interaction of Afnan and Tang, ${ }^{49}$ and the MT-V interaction of Malfliet and Tjon. ${ }^{50}$ Both potentials may be considered quasirealistic in the sense that they are a compromise between simplicity and providing a reasonable fit to the nucleon-nucleon scattering data.

Since there exist several parametrizations of these interactions, particularly in the MT-V case, with small differences, and given that modern techniques are able to give results of high precision, we specify below the particular forms used here. The Wigner part of the S3 interaction has been taken as

$$
\begin{aligned}
V_{S}(r)= & \left(1000 e^{-3 r^{2}}-163.35 e^{-1.05 r^{2}}-83 e^{-0.8 r^{2}}\right. \\
& \left.-21.5 e^{-0.6 r^{2}}-11.5 e^{-0.4 r^{2}}\right) \mathrm{MeV}
\end{aligned}
$$

where the internucleon distance $r$ is measured in units of $1 \mathrm{fm}$. The fact that this potential is a combination of Gaussian factors will allow us to carry out very precise calculations by using algebraic techniques. The MT-V potential comprises a sum of a repulsive and an attractive Yukawa term

$V_{M}(r)=\left[1458.27 \frac{e^{-3.11 r}}{r}-578.18 \frac{e^{-1.55 r}}{r}\right] \mathrm{MeV}$

with $r$ again measured in units of $1 \mathrm{fm}$. Both the S3 and MT-V potentials are considered to be fully local potentials (i.e., acting in all partial waves), and we do not include a Coulomb force between the pair of protons. In all calculations we have used a value of the nucleon mass $m$ such that $\hbar^{2} / m=41.5 \mathrm{MeV} \mathrm{fm}^{2}$.

We now briefly consider some of the technical details involved in the numerical calculations. The actual equations that we solve in the various approximation schemes described in Sec. IV are themselves relatively simple. Thus, the standard CC2 method involves the solution of a coupled set of nonlinear (actually bilinear) multinomial equations in the truncated set of coefficients $\left\{S_{n} ; n=1,2, \ldots, n_{\max }\right\}$. The remaining methods are of the basic form of generalized eigenvalue problems. Even for truncation indices $n_{\max }$ as high as the value 30 which we use here, the solution of such given sets of equations is both relatively straightforward and computationally rapid. By far the greatest part of the computational effort goes, however, into calculating the coefficients of the various terms in the equations. It is this latter task which is not a computationally trivial one, and we therefore outline the procedures that we have adopted.

Our first procedure involves solving the various cou- 
pled sets of equations not in the previous Fock-space representation, but rather in the coordinate-space representation. Furthermore, for potentials such as the S3 interaction, which consist of combinations of Gaussian terms, we have made use of a recurrence relation for the relevant matrix elements which is described in Appendix B. This procedure is very economical with respect to computer time, even when coded in quadruple precision (i.e., using 128-bit real variables). By using this level of precision it has been possible to perform $\mathrm{CI} 2$ calculations for the S3 potential up to the extremely high value of $n_{\max }=30$ (corresponding to $60 \hbar \omega$ in excitation energy) without encountering the instability problems which plague calculations at appreciably lower values of $n_{\max }$ done even in double precision. This instability arises due to the eigenvalue problem becoming unstable with regard to the compounding of small rounding errors.

These coordinate-space techniques have also permitted us to perform the comparable calculations for the S3 potential for the more ambitious CC2, CC2-E, and CI4-R approximations for values of $n_{\max } \leq 12$. In the case of the MT-V potential, not all of the coordinate-space integrals can be exactly calculated by such recursive schemes. Certain remaining integrals need to be performed numerically, and their approximate evaluation limits the accuracy attainable and the highest $n_{\max }$ that can be reached without instability problems. Nevertheless, we have found it possible to perform $\mathrm{CI} 2$ calculations for the MT-V potential in this manner up to values of $n_{\max } \approx 19$ before rounding errors again start to accumulate seriously.

Our second procedure has been to solve the equations directly in the original Fock-space representation. The formulas needed for these calculations are presented in Appendix A. We have concentrated our efforts here on the $\mathrm{CI} 2$ and $\mathrm{CC} 2$ approximations, and for both potentials we present results below for $n_{\max } \leq 29$. These very high values of $n_{\max }$ have been achieved, however, only by a considerable investment in computational effort in terms both of program development and of running time. In both of these respects, the majority of the effort has gone into the calculation of the needed Brody-Moshinsky (BM) brackets. Unfortunately, there are simply too many of these to store them all for the highest values of $n_{\max }$. On the other hand, it is fortunate that most of the actual coefficients in the $\mathrm{CI} 2$ and $\mathrm{CC} 2$ equations involve only the subset of BM brackets with a total angular momentum equal to zero. Even handling this subset still presents a formidable problem. Thus, for $n_{\max }=29$, there are approximately $5 \times 10^{5}$ distinct nonzero brackets. These were calculated once, stored, and retrieved when needed by using a modified Fibonacci hashing algorithm. ${ }^{76}$ The remaining BM brackets are too numerous to store, but fortunately are sufficiently simple to be able to be calculated as needed.

The calculations in the original Fock-space representation were performed on an Amdahl VP 1100 vector processor. This machine has an optimal vector speed of $286 \times 10^{6}$ flops. Each run for a given value of the harmonic-oscillator basis parameter $\alpha$ took $6 \mathrm{~h}$ of CPU time with $n_{\max }=29$, after a great deal of both scalar and vector optimization. The final code was $81 \%$ vectorized (with a vector efficiency rated between good and excellent). It required $30 \times 10^{6}$ bytes of real memory, $22 \times 10^{6}$ bytes of which were used for the hash table. Since this latter portion of the memory is intended to be accessed essentially randomly, it is clear that a virtual-memory machine would not have sufficed for our purposes.

The behavior of the ground-state energy $E$ as calculated in the CI2 approximation is shown in Fig. 1 with respect to the order $n_{\max }$ (i.e., where the number of basis states is $\left.n_{\max }+1\right)$. For both potentials the calculations have been performed at a fixed value of the harmonicoscillator parameter $\alpha$, namely, $0.7 \mathrm{fm}^{-1}$ for the S3 potential and $0.8 \mathrm{fm}^{-1}$ for the MT-V potential. These values correspond approximately to the values which minimize the ground-state energy estimates for the highest value of $n_{\max }=29$ used. We note that the results for the S3 potential have almost fully converged at this value of $n_{\max }$, being undoubtedly within a few tenths of 1 $\mathrm{MeV}$ of the infinite basis-size limit for this approximation. Indeed, at a value of $n_{\max }=20$ for the S3 potential, we obtain an energy estimate that is only approximately 1 $\mathrm{MeV}$ away from the fully converged limit. Conversely, even at an excitation energy of $58 \hbar \omega$, the MT-V result is still probably somewhat more than $2 \mathrm{MeV}$ away from convergence. This difference in the rate of convergence presumably stems largely from the fact that the MT-V potential has both a much more repulsive core than the $\mathrm{S} 3$ potential and an attractive tail that falls off more slowly at large separations.

In Table I we compare the $\mathrm{CI} 2$ data illustrated in Fig. 1 , with both the corresponding $\mathrm{CC} 2$ results and the essentially exact results obtained by the diffusion Monte Carlo (DMC) method. ${ }^{51}$ It is very clear that the bilinear terms which comprise the extra ingredient in the CC2 method contribute only a few tenths of $1 \mathrm{MeV}$ additional binding

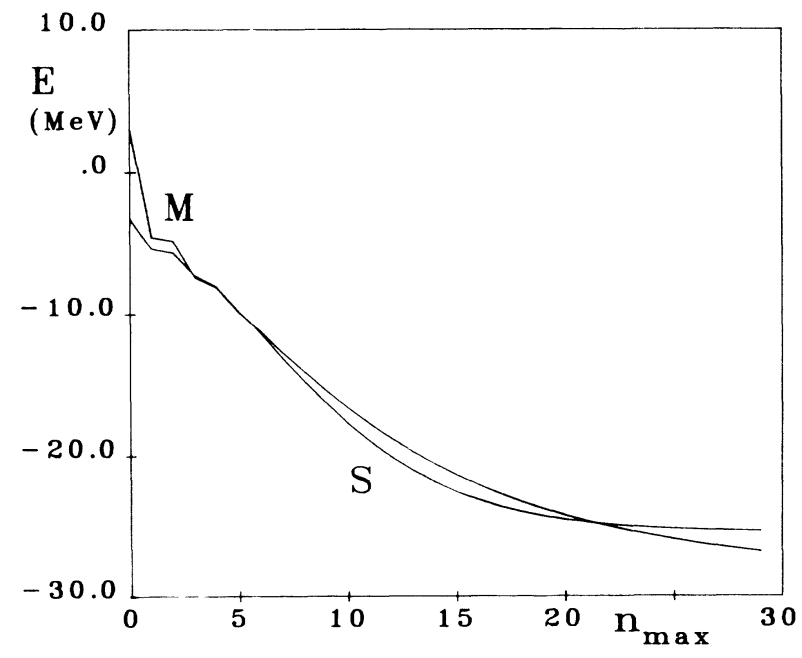

FIG. 1. The ground-state energy of the ${ }^{4} \mathrm{He}$ nucleus calculated in the CI2 approximation as a function of the order $\left(n_{\max }\right)$ of truncation, for the $\mathrm{S} 3$ potential $(S)$ with $\alpha=0.7 \mathrm{fm}^{-1}$ and the MT-V potential $(M)$ with $\alpha=0.8 \mathrm{fm}^{-1}$, where $\alpha$ is the oscillator parameter of the single-particle basis. 
TABLE I. Results for the ground-state energy in $\mathrm{MeV}$ for ${ }^{4} \mathrm{He}$ as a function of the basis size, calculated via the $\mathrm{CC} 2$ and CI2 approximations described in the text, for the S3 and MT-V potentials, with the basis parameter $\alpha$ set to 0.7 and $0.8 \mathrm{fm}^{-1}$, respectively. Also shown are the essentially exact DMC results from Ref. 51.

\begin{tabular}{|c|c|c|c|c|}
\hline \multirow[b]{2}{*}{$n_{\max }$} & \multicolumn{2}{|c|}{$\mathrm{S} 3$} & \multicolumn{2}{|c|}{ MT-V } \\
\hline & $\mathrm{CI} 2$ & $\mathrm{CC} 2$ & $\mathrm{CI} 2$ & $\mathrm{CC} 2$ \\
\hline 0 & -3.250 & & 9.512 & \\
\hline 1 & -5.357 & -5.379 & -3.347 & -3.640 \\
\hline 2 & -5.637 & -5.671 & -3.629 & -3.947 \\
\hline 3 & -7.275 & -7.367 & -7.104 & -7.696 \\
\hline 4 & -8.080 & -8.159 & -7.981 & -8.507 \\
\hline 5 & -9.795 & -9.901 & -9.997 & -10.590 \\
\hline 6 & -11.364 & -11.464 & -11.476 & -12.024 \\
\hline 7 & -13.101 & -13.211 & -13.117 & -13.665 \\
\hline 8 & -14.735 & -14.847 & -14.562 & -15.080 \\
\hline 9 & -16.289 & -16.407 & -15.941 & -16.441 \\
\hline 10 & -17.695 & -17.818 & -17.181 & -17.657 \\
\hline 11 & -18.952 & -19.081 & -18.315 & -18.771 \\
\hline 12 & -20.050 & -20.186 & -19.331 & -19.768 \\
\hline 13 & -20.997 & -21.140 & -20.246 & -20.665 \\
\hline 14 & -21.803 & -21.952 & -21.064 & -21.468 \\
\hline 15 & -22.482 & -22.636 & -21.797 & -22.186 \\
\hline 16 & -23.048 & -23.207 & -22.451 & -22.827 \\
\hline 17 & -23.515 & -23.678 & -23.035 & -23.401 \\
\hline 18 & -23.899 & -24.065 & -23.558 & -23.913 \\
\hline 19 & -24.210 & -24.380 & -24.026 & -24.372 \\
\hline 20 & -24.462 & -24.634 & -24.445 & -24.783 \\
\hline 21 & -24.664 & -24.837 & -24.820 & -25.151 \\
\hline 22 & -24.824 & -24.999 & -25.158 & -25.482 \\
\hline 23 & -24.951 & -25.127 & -25.461 & -25.780 \\
\hline 24 & -25.051 & -25.228 & -25.734 & -26.048 \\
\hline 25 & -25.128 & -25.306 & -25.981 & -26.290 \\
\hline 26 & -25.188 & -25.366 & -26.205 & -26.509 \\
\hline 27 & -25.234 & -25.412 & -26.408 & -26.708 \\
\hline 28 & -25.269 & -25.447 & -26.595 & -26.891 \\
\hline 29 & -25.294 & -25.473 & -26.769 & -27.062 \\
\hline DMC & \multicolumn{2}{|c|}{$-26.9 \pm 0.2$} & \multicolumn{2}{|c|}{$-31.5 \pm 0.2$} \\
\hline
\end{tabular}

TABLE II. Results for the ${ }^{4} \mathrm{He}$ ground-state energy in $\mathrm{MeV}$ as a function of the basis size, calculated in the various approximations discussed in the text, for the $\mathrm{S} 3$ potential and with the basis parameter $\alpha=0.7 \mathrm{fm}^{-1}$.

\begin{tabular}{rccr}
\hline$n_{\max }$ & $\mathrm{CC} 2$ & $\mathrm{CC} 2-\mathrm{E}$ & $\mathrm{CI} 4-\mathrm{R}$ \\
\hline 1 & -5.379 & -5.394 & -5.432 \\
2 & -5.671 & -5.693 & -5.761 \\
3 & -7.367 & -7.424 & -7.529 \\
4 & -8.159 & -8.202 & -8.281 \\
5 & -9.901 & -9.954 & -10.061 \\
6 & -11.464 & -11.505 & -11.581 \\
7 & -13.211 & -13.248 & -13.321 \\
8 & -14.847 & -14.875 & -14.930 \\
9 & -16.407 & -16.429 & -16.476 \\
10 & -17.818 & -17.833 & -17.870 \\
11 & -19.081 & -19.092 & -19.121 \\
12 & -20.186 & -20.193 & -20.216 \\
\hline \hline
\end{tabular}

energy for both potentials investigated and for large values of $n_{\max }$ at values of $\alpha$ near that which minimizes the energy. The precise value of the difference in binding energies obtained by the two methods depends (in a rather complicated fashion) on the order $n_{\max }$ but the difference is always small. We note that the CI2 approximation for the $\mathrm{S} 3$ potential already by itself gives about $95 \%$ of the total binding energy. Of the missing $5 \%$, the extra bilinear terms in the $\mathrm{CC} 2$ approximation, which represent the independent excitation of two $2 \mathrm{p}-2 \mathrm{~h}$ pairs, contribute only a relatively small fraction. This result is further reinforced in Table II where the CC2 results are compared with the variational upper bound results of the corresponding CC2-E calculations. We note, in particular, that for all basis sizes used in this comparison, $n_{\max } \leq 12$, the $\mathrm{CC} 2$ and $\mathrm{CC} 2-\mathrm{E}$ results are within a few hundredths of $1 \mathrm{MeV}$ of each other, thereby providing a good internal consistency check on the CCM approximations.

In Table II we also show the corresponding results for the S3 potential with the CI4-R approximation. A comparison of the two variational results from the CC2-E and CI4- $R$ approximations indicates that the enlargement of the variational space to include the restricted class of $4 \mathrm{p}$ $4 \mathrm{~h}$ excitations admitted by the latter approximation, results in a lowering of the energy estimate by less than about $0.1 \mathrm{MeV}$. It is clear from all of these results that the CI 2 calculation does very well indeed by itself (once it has converged), and furthermore that the binding energy that it misses is difficult to attain by any of the other means discussed. These same general features are also displayed by the MT-V potential, with the main difference being the slower convergence. Nevertheless, even for this potential, the fully converged CI 2 approximation almost certainly yields over $90 \%$ of the total binding energy.

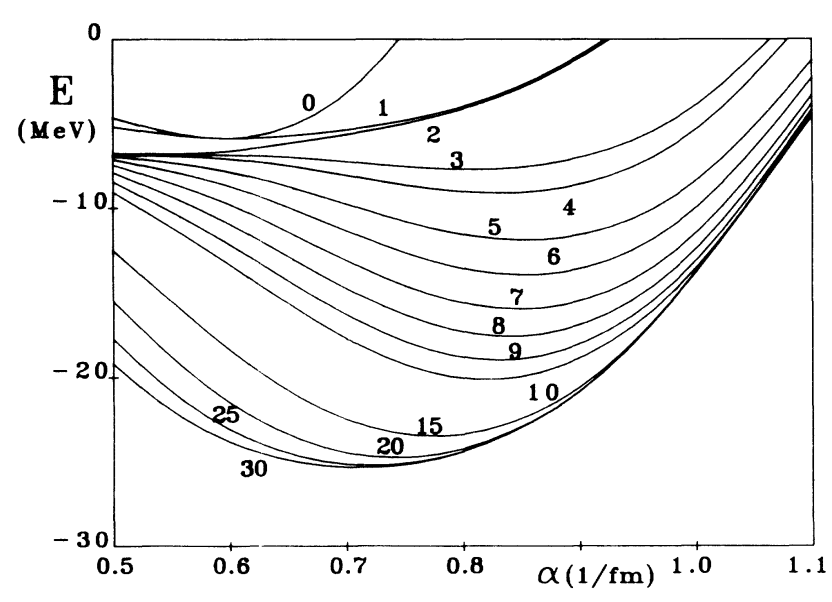

FIG. 2. The ground-state energy of the ${ }^{4} \mathrm{He}$ nucleus calculated with the $\mathrm{S} 3$ potential and at various levels of basis truncation in the CI2 approximation, as a function of the oscillator parameter $\alpha$ which characterizes the single-particle basis. The different curves are labeled by the respective order $\left(n_{\max }\right)$ of the truncation. 
We turn now to the question of the role of tine harmonic-oscillator constant $\alpha$ in the convergence of the results. In Fig. 2 we examine the ground-state energy of the S3 potential as a function of the parameter $\alpha$ for different values of the basis size in a CI 2 calculation. These data display several strong features which are not easily intuited. In particular, they demonstrate both the very complex nature of the convergence process, and the critical importance of the choice of parameter $\alpha$, even at very large values of the basis size. Furthermore, the optimal choice of $\alpha$ to minimize the energy is seen to be quite sensitive to the choice of $n_{\max }$. Indeed the optimal value of $\alpha$ only stabilizes after a large value of $n_{\max }$ is reached.

Perhaps even more importantly, one observes from Fig. 2 several examples of regions of extremely nonuniform convergence and of seeming, but false, convergence. Thus, for the fixed value of $\alpha$ of about $0.5 \mathrm{fm}^{-1}$, the CI2 calculation gives every indication of converging to the very false value of about $-7 \mathrm{MeV}$ for basis sizes up to $n_{\max }=5$, before the calculation "depins" from this value as $n_{\max }$ is increased further. Even more seriously, for values $\alpha \gtrsim 0.95 \mathrm{fm}^{-1}$, the CI2 calculations appear completely converged for values of $n_{\max }$ as high as 30 , and probably even much higher, although to quite the wrong minimal value. Although in a full CI or CCM calculation, the results must be independent of the original model state, and hence of $\alpha$, the limit $n_{\max } \rightarrow \infty$ of our CI2 calculation need not be wholly independent of $\alpha$ since we have neglected $3 \mathrm{p}-3 \mathrm{~h}$ and $4 \mathrm{p}-4 \mathrm{~h}$ excitations. Naively, we might expect, however, that as $n_{\max }$ is increased the CI2 energy estimate would smoothly become more independent of $\alpha$ as the large basis limit is approached. Curiously, the calculations with $n_{\max } \approx 4$ are appreciably less dependent on $\alpha$ than those with $n_{\max } \approx 30$, even though the former give extremely poor estimates for the energy eigenvalue.

The same general behavior is also true for the MT-V potential, although it presents a greater challenge to our techniques in view of the overall slower pattern of convergence. In view of the very complex nature of the convergence process, it is clear that any attempt to extrapolate the CI2 results from our highest basis size $n_{\max }=29$ to the infinite limit will be fraught with danger at whatever value of $\alpha$ is chosen. While this is undoubtedly the case, all such attempts indicate that the CC2 estimate for the energy is unlikely to converge to a greater value of the binding energy than about $29.5 \mathrm{MeV}$. Once again, the remaining $2 \mathrm{MeV}$ or so must be due to the neglect of three- and four-body correlations. The detailed dependence on the harmonic-oscillator constant $\alpha$ of both the $\mathrm{CI} 2$ and CC2 results for the MT-V potential is displayed in Fig. 3. We observe again the very flat curves for $n_{\text {max }} \approx 5$, and the possibility that exists if one had only data for bases of up to this size, to make the natural, but false, assumption that the energy estimates are (nearly) converged, especially for the lower values of $\alpha$ shown. It is also worth pointing out that the nonlinear terms in the CC2 approximation produce a non-negligible lessening of the dependence on $\alpha$, though, as noted above, we see that near the large- $n_{\max }$ energy minimum they have little

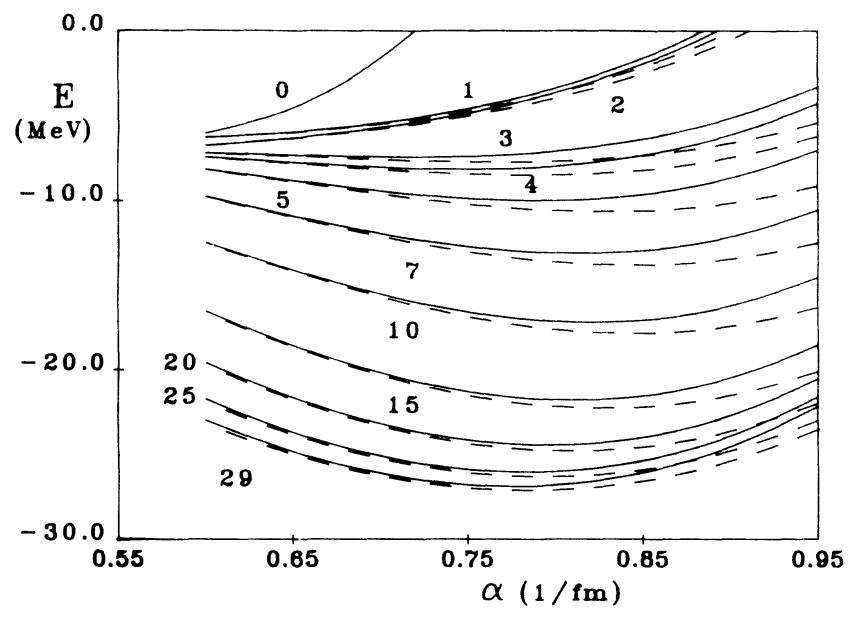

FIG. 3. The ground-state energy of the ${ }^{4} \mathrm{He}$ nucleus calculated with the MT-V potential and at various levels of basis truncation in the CI2 approximation (solid lines) and the CC2 approximation (dashed lines), as a function of the oscillator parameter $\alpha$ which characeterizes the single-particle basis. The different curves are labeled by the order $\left(n_{\max }\right)$ of the truncation.

effect.

We now turn to an examination of the coordinatespace representation of our CI2 wave function. It is clear from either Eq. (23) or Eq. (30) that this may be written in the form

$$
\left.\left\langle\mathbf{r}_{1} \mathbf{r}_{2} \mathbf{r}_{3} \mathbf{r}_{4} \mid \Psi_{\mathrm{Cl} 2}\right\rangle=\mid \sum_{i<j=1}^{4} f\left(r_{i j}\right)\right] \exp \left(-\frac{1}{2} \alpha^{2} \sum_{k=1}^{4} r_{k}^{2}\right),
$$

where $f(r)$ is a polynomial in $r^{2}$ of order $n_{\max }$, which is determined from the eigenvectors of the eigenvalue problem described above in Sec. IV. For convenience, we now choose the normalization of the approximate wave function so that the correlation factor $f(r)$ takes the value unity at the origin $f(0)=1$ for the potentials which are themselves finite at the origin, like the S3 potential. Figure 4 illustrates a plot of the CI2 correlation factor $f(r)$ for the $\mathrm{S} 3$ potential for various values of the basis-size parameter $n_{\max }$. We note the clear convergence, at least for the range of values of the separation distance $r$ shown, as $n_{\max }$ is increased. Clearly, for large distances $r$, the function $f(r)$ will become infinite for any value of $n_{\max }$ due to its finite-polynomial nature. This behavior is not dangerous, since the Gaussian factor in Eq. (35) will kill any such polynomial growth, and the proper asymptotic behavior of the wave function may thereby be restored.

Finally, for purposes of comparison we consider the analogous implementation of the above approximations wholly within the coordinate representation. In the first place, we may seek the form of the correlation factor $f(r)$ in Eq. (35) which minimizes the energy expectation value over the CI2 class of trial wave functions. The solution of this problem is wholly equivalent to the CI2 approximation. Rather than attempting a direct solution of the resulting Euler-Lagrange equation, a very con- 
venient and efficient method is to parametrize the function $f(r)$ approximately as a linear combination of Gaussian functions of different ranges,

$$
f(r) \simeq \sum_{n=1}^{N} A_{n} \exp \left(-b_{n} r^{2}\right)
$$

and to seek the minimum of the resulting energy expectation value over the set of variational parameters $\left\{\alpha, b_{n}, A_{n}\right\}$. If the set of nonlinear parameters $\left\{\alpha, b_{n}\right\}$ is first specified, the problem of then determining the linear parameters $\left\{A_{n}\right\}$ is a straightforward generalized eigenvalue problem. The main part of the search for the minimum thus involves the set $\left\{\alpha, b_{n}\right\}$. As is well known, the nonorthogonality of the basis in Eq. (36) leads to the minimization problem rather easily becoming ill conditioned if the values of the parameters $\left\{b_{n}\right\}$ become too close to each other. Thus, one should not attempt to use too large or too dense a Gaussian basis.

It is clear from Eq. (30) and from our previous discussion that the comparable CI4-R analysis is also quite similar. The coordinate-space wave function is now approximated as

$$
\left\langle\mathbf{r}_{1} \mathbf{r}_{2} \mathbf{r}_{3} \mathbf{r}_{4} \mid \Psi_{\mathrm{CI} 4-\mathbf{R}}\right\rangle \simeq \sum_{n, n^{\prime}=1}^{N} A_{n n^{\prime}} \sum_{i<j=1}^{4} \exp \left(-b_{n} r_{i j}^{2}-b_{n^{\prime}} r_{k l}^{2}\right) \exp \left(-\frac{1}{2} \alpha^{2} \sum_{m=1}^{4} r_{m}^{2}\right),
$$

in terms of a set of parameters $\left\{b_{n}\right\}$ which includes the value zero, so that both the one- and two-pair terms in Eq. (30) are now represented, and where both indices $k$ and $l$ are different from both indices $i$ and $j$, as before. Once again, the CI4-R approximation results in a generalized eigenvalue problem for the set of amplitudes

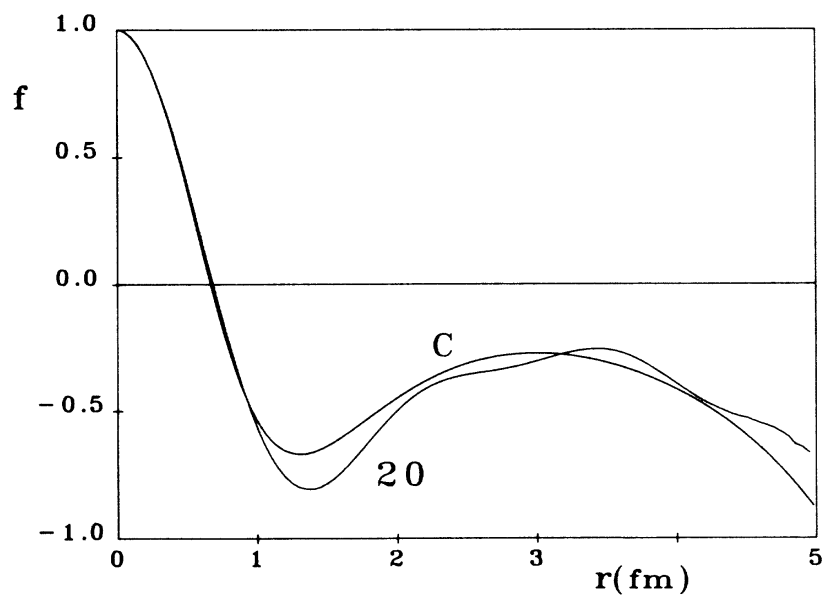

FIG. 5. The correlation factor $f(r)$ that characterizes the $\mathrm{CI} 2$ approximation to the ${ }^{4} \mathrm{He}$ ground-state wave function via the parametrization of Eq. (35) and using the S3 potential. The curve labeled 20 corresponds to the $n_{\max }=20$ curve in the truncated oscillator representation of Fig. 4 with $\alpha=0.7 \mathrm{fm}^{-1}$, while the curve labeled $C$ corresponds to the coordinate-space representation of Eq. (36) with $N=10, \alpha=0.71 \mathrm{fm}^{-1}$, and $\left\{b_{n}\right\}$ values as given in the text.

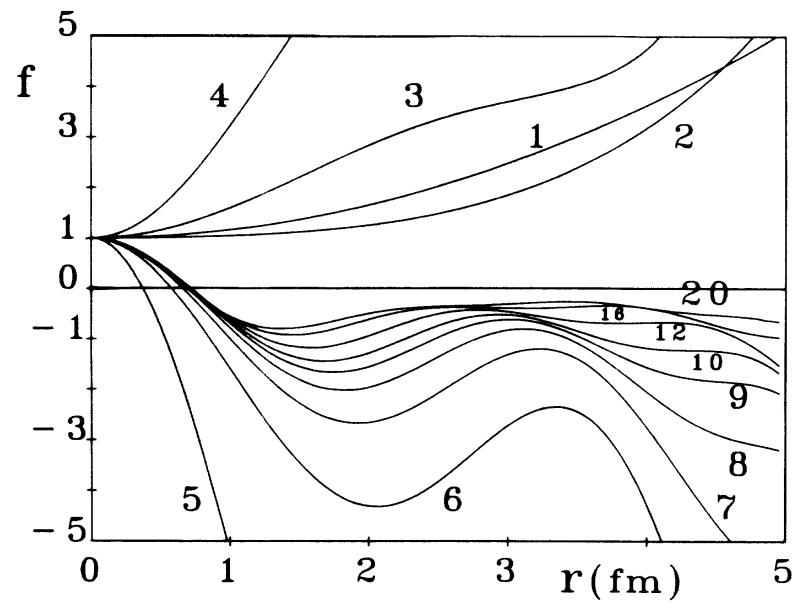

FIG. 4. The correlation factor $f(r)$ that characterizes the CI2 approximation to the ${ }^{4} \mathrm{He}$ ground-state wave function via the parametrization of Eq. (35). The calculation employs the S3 potential and a value $\alpha=0.7 \mathrm{fm}^{-1}$ for the oscillator parameter of the single-particle basis. The different curves are labeled by the respective order $\left(n_{\max }\right)$ of the truncation.
$\left\{A_{n n^{\prime}}\right\}$ for fixed values of the set $\left\{\alpha, b_{n}\right\}$.

Using a set of values $\left\{b_{n}\right\}=\{-0.08,0,0.35,1,2,4,8$, $16,32,64 \mathrm{fm}^{-2}$, we obtain CI2 and CI4-R estimates for the ground-state energy of -25.36 and $-25.55 \mathrm{MeV}$, respectively, for the $\mathrm{S} 3$ potential, and -29.40 and -29.58 $\mathrm{MeV}$, respectively, for the MT-V potential. These approximately minimal values are attained in each case with oscillator parameter $\alpha=0.71 \mathrm{fm}^{-1}$ for the S3 potential and $\alpha=0.74 \mathrm{fm}^{-1}$ for the MT-V potential. We believe that these approximately minimal energy eigenvalues are very close to the precise results that pertain to each level of approximation, since, by making further changes in any of the variational parameters or by making further increases in the basis size $N$, we are unable appreciably to lower these estimates any further. In Fig. 5 we compare, for the $\mathrm{S} 3$ potential, the corresponding CI2 correlation factors $f(r)$ from the oscillator basis representation and from the present coordinate-space basis representation of Eq. (36) with $N=10$ and with the set $\left\{b_{n}\right\}$ as given above.

\section{DISCUSSION AND COMPARISON}

It is of interest to compare and contrast our own CI and CCM calculations reported here with the more standard shell-model calculations. These latter calculations are usually performed in a so-called complete space which includes all possible configurations with a bare (noninteracting) excitation energy up to a given maximal value $N \hbar \omega$ in order to guarantee no spuriosity associated with the center-of-mass motion. Thus, in such complete 
spaces, it is known that it is possible to construct a basis in which every state is an eigenstate of the center-of-mass Hamiltonian. ${ }^{74}$ The problem, in practice, with such complete model spaces is that their dimensionality grows alarmingly rapidly with the truncation index $N$. Furthermore, a CI calculation in such an energy-truncated basis leads to a replication of the approximated intrinsic states, with each possible center-of-mass eigenstate being separately associated with each intrinsic state attainable within the overall constraint on the maximum excitation energy. One of the most important findings of the present paper is that it is perfectly possible to construct many-particle bases in which the number of configurations, constrained to $1 \mathrm{p}-1 \mathrm{~h}$ and $2 \mathrm{p}-2 \mathrm{~h}$ excitations, is dramatically decreased for a given value of $N$, but wherein the center-of-mass motion is again always properly factorizable. Indeed, it is actually always in the lowest $(0 s)$ state.

Furthermore, we have seen that these translationally invariant $\mathrm{CI}$ and CCM calculations, built from the harmonic-oscillator single-particle basis and incorporating only one- and two-body correlations, are capable, in practice, of obtaining approximately $90-95 \%$ of the total binding energy of the ${ }^{4} \mathrm{He}$ nucleus for the quasirealistic potentials used. The sole, but important, proviso is that the calculations must be performed in a large enough truncated basis to reach convergence. We have clearly observed the necessity to use bases which include very high values of the oscillator excitation energy in order to attain convergence. Values of $N \approx 60$ are necessary for the S3 potential for the energy estimate to have converged to a few tenths of $1 \mathrm{MeV}$. Appreciably higher values still are needed for the MT-V potential to reach the same degree of convergence. To include such states in calculations involving complete spaces would involve such enormously large numbers of configurations as to be computationally quite impracticable to handle.

Before we compare our numerical results with those of other calculations, we shall attempt to understand why the convergence of our translationally invariant $\mathrm{CI}$ and CCM calculations is so slow with respect to the truncation index $n_{\max }$. The first point to note is that an $A$-body wave function of the form given in Eq. (35) obeys the cluster property in the following sense. Suppose that one of the $A$ particles, say, particle 1 at position $\mathbf{r}_{1}$, is moved far away from the remaining $(A-1)$ particles, whose center of mass is at the position

$$
\mathbf{R}^{\prime}=\sum_{i=2}^{A} \mathbf{r}_{i} /(A-1) .
$$

Thus, in the limit as $r_{1}^{\prime} \equiv\left|\mathbf{r}_{1}-\mathbf{R}^{\prime}\right| \rightarrow \infty$, the intrinsic part of the Gaussian factor in Eq. (35) will decrease like

$$
\exp \left[-\frac{1}{2} r_{1}^{\prime 2} \alpha^{2}(A-1) / A\right] \text {, }
$$

once the center-of-mass wave function has been removed by use of Eqs. (12)-(14). Given the polynomial nature of the correlation factors in the truncated $\mathrm{CI} 2$ calculations, the correlation part of Eq. (35) will increase in the same limit as $(A-1) f\left(r_{1}^{\prime}\right)$. The polynomial growth of this last factor is more than compensated by the exponential decay of the uncorrelated wave function. The product of the two factors thus approaches zero whenever any one of the particles becomes far removed from the remainder.

On the other hand, in an exact treatment of this dissociation limit, one expects the wave function to decrease exponentially, i.e., as $\exp \left(-\varepsilon r_{1}^{\prime}\right)$, where $\varepsilon$ is proportional to the dissociation energy to remove one particle from the $A$-particle nucleus. Undoubtedly, it will be necessary to include many terms in the power series expansion for $f(r)$ before the correlation factor can correctly compensate for the incorrect limiting behavior of the uncorrelated Gaussian wave function. In conclusion, the form of $f(r)$ as a polynomial in $r^{2}$ is simply not very appropriate to reproduce accurately the long-range behavior, and hence many terms are needed. Of course, this basic form for $f(r)$ stems ultimately from the basic harmonicoscillator form of the uncorrelated wave function or, equivalently, from our choice of single-particle orbitals to characterize the Fock space. Furthermore, this choice of harmonic-oscillator orbitals was motivated by the desire to remove easily and exactly any spuriosity associated with the motion of the center of mass.

From the above considerations, we observe that while the harmonic-oscillator basis is an extremely convenient one for purposes of handling the center-of-mass motion, its main disadvantage is that one has to include configurations of very high excitation energy in order to reach convergence or, equivalently, in order to reproduce accurately the large-distance behavior of the many-body wave function in the dissociation limit. From such general observations and from our own numerical investigations, one is then forced to conclude that such an expansion in the intrinsic oscillator wave functions is not necessarily the most sensible way to handle the various CI and CCM equations at any of the several levels of approximate implementation of these methods. From this viewpoint it is particularly interesting to compare our results both with the corresponding CCM calculation of Zabolitzky ${ }^{8}$ for the ${ }^{4} \mathrm{He}$ nucleus which also employed the MT-V potential among others, and with such more traditional shell-model calculations of the same system as those of Ceuleneer et al. ${ }^{54}$ who employed a complete basis space of configurations up to $10 \hbar \omega$ in excitation energy.

The CCM calculation of $\mathrm{Zabolitzky}^{8}$ is based not on our own translationally invariant basis discussed here, but rather on the more usual CCM approach of handling the center-of-mass motion, which has been enunciated by Fink. ${ }^{77}$ The aim is to work directly with the internal or intrinsic Hamiltonian $H_{\text {int }} \equiv H-T_{\text {c. m. }}$ and to solve for its corresponding ground-state wave function $\left|\Psi_{\text {int }}\right\rangle$ in the usual CCM form,

$$
\left|\Psi_{\text {int }}\right\rangle=\exp \left(S_{\text {int }}\right)\left|\Phi_{\text {int }}\right\rangle
$$

where $\left|\Psi_{\text {int }}\right\rangle$ and $\left|\Phi_{\text {int }}\right\rangle$ are now functions of some suitable set of $(A-1)$ internal coordinates, $\xi_{1}, \ldots, \xi_{A-1}$. As usual, the difficulty in obtaining $\left|\Psi_{\text {int }}\right\rangle$ is that the coordinates are not treated symmetrically. The same simple remedy is adapted as in our own work, namely, the use of harmonic-oscillator wave functions for the many-body model state $\left|\Phi_{\text {int }}\right\rangle$. A simple multiplication 
by the oscillator wave function $\left\langle\mathbf{R} \mid \Phi_{\text {c.m. }}\right\rangle$ for the ground state of the center of mass then leads, for doubly magic nuclei, to the form

$$
\left\langle\xi_{1} \ldots \xi_{A-1} \mid \Psi_{\text {int }}\right\rangle\left\langle\mathbf{R} \mid \Phi_{\text {c.m. }}\right\rangle=\exp \left(S_{\text {int }}\right)\left\langle\mathbf{r}_{1} \ldots \mathbf{r}_{A} \mid \Phi\right\rangle \text {, }
$$

where $|\Phi\rangle$ is a Slater determinant of oscillator wave functions [or the simple product of Eq. (12) in our own bosonic case for ${ }^{4} \mathrm{He}$ with Wigner forces]. The corresponding eigenvalue equation is

$$
\left(H_{\text {int }}-E_{\text {int }}\right) \exp \left(S_{\text {int }}\right)\left\langle\mathbf{r}_{1} \ldots \mathbf{r}_{A} \mid \Phi\right\rangle=0,
$$

and the exact wave function in a state where the c.m. has momentum $\mathbf{K}$ is

$$
\left\langle\mathbf{r}_{1} \ldots \mathbf{r}_{A} \mid \Psi\right\rangle=\frac{\exp \left(S_{\text {int }}\right)\left\langle\mathbf{r}_{1} \ldots \mathbf{r}_{A} \mid \Phi\right\rangle}{\left\langle\mathbf{R} \mid \Phi_{\text {c. m. }}\right\rangle} V^{-1 / 2} e^{i \mathbf{K} \cdot \mathbf{R}},
$$

where $V$ is the normalization volume.

In the standard procedure outlined above, the $1 \mathrm{p}-1 \mathrm{~h}$ excitations have to be calculated explicitly via the onebody partition $S_{\text {int, } 1}$ of the cluster operator $S_{\text {int }}$. Furthermore, the treatment of the c.m. motion is exact only as long as the CCM parametrization is not truncated. Otherwise, its treatment is as accurate as the particular $\operatorname{SUB}(n)$ truncation used. Zabolitzky ${ }^{8}$ has performed calculations at the SUB(2), SUB(3), and SUB(4) levels for the ${ }^{4} \mathrm{He}$ nucleus with the MT-V potential. At the SUB(2) level, his doubly self-consistent numerical treatment of the one- and two-body equations was essentially exact, whilst, at the SUB(3) level, some small inhomogeneous terms in the resulting three-body Bethe-Faddeev equation were neglected. At the SUB(4) level, the solution of the relevant four-body equation was no longer feasible, and only the factorizable contributions from this equation and those omitted in the three-body equation were included.

At the SUB(2) level, where the c.m. treatment is not exact, no constraints are imposed on the matrix elements of the one- and two-body cluster operators, in contrast to what is done in our own treatment. Furthermore, and very importantly for comparison purposes, although the analysis was based in the first place on an oscillator wave function basis for the Fock space, the two-body equation was actually solved in coordinate space by a further transformation from the intrinsic oscillator basis $|n l\rangle$ to the relative coordinate basis $|r l\rangle$. In this way the convergence problem is side-stepped. By this procedure, Zabolitzky obtained a SUB(2) estimate for the ground-state energy of ${ }^{4} \mathrm{He}$ with the MT-V potential of $-30.24 \mathrm{MeV}$. This may be compared in the first place with our own CC2 results in Table I, which give $-27.06 \mathrm{MeV}$ at $n_{\max }=29$, but which are not yet fully converged. It may perhaps also be more closely compared with our own coordinate-space CI4-R variational result of -29.58 $\mathrm{MeV}$, which we believe is close to the precise (converged) values for both this approximation and, from the results of Table II, the CC2 approximation.

The remaining difference of approximately $0.6-0.7$
$\mathrm{MeV}$ is due to the inclusion by Zabolitzky of a selfconsistent $1 \mathrm{p}-1 \mathrm{~h}$ cluster operator (and the corresponding inaccuracy in handling the c.m. motion). In our own calculations, such effects would be subsumed into the omitted $3 p-3 h$ and $4 p-4 h$ correlations. At SUB(3) and SUB(4) levels, Zabolitzky finds the corresponding energy estimates of -31.24 and $-31.36 \mathrm{MeV}$, but in view of the inherent approximations in the latter calculation, the best estimate is probably the SUB(3) result. In any case, both of these latter estimates are gratifyingly close to our own essentially exact DMC result ${ }^{51}$ of $-31.5 \pm 0.2 \mathrm{MeV}$, and indeed also to an earlier comparable Green's-function Monte Carlo (GFMC) result ${ }^{78}$ of $-31.3 \pm 0.2 \mathrm{MeV}$.

We turn next to a comparison with the more traditional shell-model (CI) calculations. Typical of the most ambitious of these is the work of Ceuleneer et al. ${ }^{54}$ These authors have performed calculations for ${ }^{4} \mathrm{He}$ with the MT-V potential, using complete model spaces of total excitation energy $N \hbar \omega$ with $N \leq 10$. The numbers of fourparticle configurations involved with $N=2,4,6,8$, and 10 are $7,45,221,853$, and 2765 , respectively. Their corresponding results for the ground-state energy, optimized for the choice of oscillator constant $\alpha$ for each value of $N$, are $-6.40,-7.29,-8.28,-13.77$, and $-18.31 \mathrm{MeV}$, respectively. These results are not immediately comparable with our corresponding results in Table I for the values $n_{\max }=1, \ldots, 5$ since our own results are shown at a fixed value of $\alpha$. A closer comparison with Fig. 2 of Ref. 54, which shows the estimates at each value of $N$ as a function of $\alpha^{-1}$, does, however, indicate that their results lie below ours, at the same value of $\alpha$, as they should do (since they also include some $3 \mathrm{p}-3 \mathrm{~h}$ and $4 \mathrm{p}-4 \mathrm{~h}$ configurations), but not by a very large amount. In any case, it is clear that even at the optimized values, their results up to $10 \hbar \omega$ in excitation energy are both far from convergence and far from the exact result, as would be expected from our own work. Similar results and conclusions have been obtained by other authors (see, e.g., Ref. 79), although typically from calculations which have not converged.

\section{CONCLUSIONS}

The starting point of our discussion has been to build the ${ }^{4} \mathrm{He}$ ground-state correlations on top of the uncorrelated four-body model state $\left|(0 s)^{4}\right\rangle$ formed from singleparticle oscillator states. The strict incorporation of translational invariance at the level where all independent ( $1 \mathrm{p}-1 \mathrm{~h}$ plus) $2 \mathrm{p}-2 \mathrm{~h}$ excitations are included, has led to the $\mathrm{CC} 2$ form of Eq. (30) as our approximate form for the ground-state wave function in coordinate space. By starting with this ansatz, the unknown two-body cluster correlation function $h(r)$ may, in principle, then be determined in several different ways.

In the first place, the standard CCM machinery may be utilized to write down an integro-differential equation for $h(r)$ following similar lines to the SUB(2) calculation of Zabolitzky $^{8}$ which gave a value of $-30.24 \mathrm{MeV}$ for the ${ }^{4} \mathrm{He}$ ground-state energy using the MT-V potential, but which did not exactly incorporate translational invariance. Secondly, one could derive an Euler-Lagrange 
equation for $h(r)$ by a functional minimization of the energy expectation value along very similar lines to the comparable calculation of Bracci et al. ${ }^{80}$ These latter authors employed a Jastrow correlated trial form for the wave function, $\Pi_{i<j} f_{J}\left(r_{i j}\right)$, and thereby obtained a minimal value of $-31.35 \mathrm{MeV}$ for the ground-state energy using the MT-V potential It is particularly interesting to note that, by writing $f_{J}(r) \equiv 1+h_{J}(r)$, our own Eq. (30) is of precisely the same form as is obtained by expanding the Jastrow correlation factor $h_{J}(r)$, and, furthermore, keeping only those (disconnected) second-order terms which relate to two independent pairs. Thirdly, the function $h(r)$ could also be determined by a method analogous to the method of hyperspherical harmonics, ${ }^{81,82}$ which has given a value between -31.22 and -30.48 $\mathrm{MeV}$ for the ground-state energy of the ${ }^{4} \mathrm{He}$ nucleus with the MT-V potential. Indeed, the corresponding CI 2 form of our wave function in which the bilinear term in $h(r)$ is omitted from Eq. (30), is very similar to that used in hyperspherical harmonic theory, as may be seen by a comparison with Eq. (5) of Ref. 82.

For the present purposes, we have mostly chosen not to work directly with the cluster correlation function $h(r)$ itself, but rather with its decomposition in terms of the oscillator basis. Thus, we have put most effort into solving directly for the amplitudes $\left\{S_{n}\right\}$ in Eq. (26) or Eq. (28), in order to make comparisons with and draw implications for other generalized shell-model calculational schemes. In particular, our own exact treatment of translational invariance has enabled us to reduce the size of the many-body oscillator configuration space so dramatically that we could perform calculations up to the very high level of $60 \hbar \omega$ excitation energy.

The four main conclusions of our work may be summarized as follows. Firstly, we have shown very clearly that, at least for energy calculations, the most important part of the wave function of the ${ }^{4} \mathrm{He}$ nucleus may be expressed in the form

$$
\sum_{i<j} f\left(r_{i j}\right)\left|(0 s)^{4}\right\rangle .
$$

Its use corresponds both to a configuration-interaction calculation in a restricted $2 \mathrm{p}-2 \mathrm{~h}$ subspace which incorporates translational invariance, and to the corresponding linearized version of translationally invariant coupled cluster theory at the SUB(2) level of approximation. This form of the wave function is also both very similar to that used in the hyperspherical harmonics approximation, and equivalent to a small correlation expansion of the Jastrow counterpart. We may thereby understand how and why all of these calculational schemes provide such good descriptions of such light systems as the ${ }^{4} \mathrm{He}$ nucleus.

Secondly, we have demonstrated that the coordinatespace methods are computationally much more efficient than the corresponding oscillator-basis expansions in attaining convergence to comparable levels of accuracy.

It has long been clear that the CCM provides one of the most efficient and systematic ways of incorporating the multiparticle correlations beyond the various paircorrelation approximation methods described above.
Our third conclusion is that the errors made in the earlier standard implementations of the CCM, which do not exactly incorporate translational invariance at the various $\mathrm{SUB}(n)$ levels of truncation, are small. We have clearly seen, for example, how the errors at the SUB(2) level are no worse than those made in neglecting the three- and four-body clusters.

By contrast with the above main points, we have stressed the calculations in the harmonic-oscillator basis in view of what they imply for standard shell-model calculations, even the biggest of which are generally only able to include configurations with much lower excitation energy than those handled by us in the present work. These implications are striking and clear. They form our fourth and final main conclusion. Thus, it is certainly a perfectly valid approximate procedure to use an effective interaction, fitted to a number of nuclear properties, in a fixed and highly restricted shell-model space. However, there is no theoretical basis then to enlarge this space to higher-energy shell-model configurations without readjusting the effective interaction at the same time. The logical endpoint of this procedure is clearly to use the bare microscopic internucleon force and no curtailment of the many-body configuration space, except that permitted by considerations of convergence. What we have seen only too clearly here is that this convergence is then so disappointingly slow as to bring the whole procedure into question.

It is apparent that there is little or no future in pursuing the translationally invariant calculations described here in the oscillator representation, due to the extremely slow convergence. The preferred future strategy for the inclusion of translational invariance is clearly to concentrate on the cluster correlation function in its coordinate-space representation $h(r)$. We also intend to calculate $h(r)$ directly by solving the resulting appropriate Euler-Lagrange equation. The approximate CI 2 and CI4-R calculations, in which the minimization is performed instead with respect to the (nonlinear) parameters of some predetermined form (e.g., a linear combination of Gaussian functions), have also been seen to be sufficiently simple and accurate to consider extending for the inclusion of three-body correlations via the cluster operator $S^{(3)}$. Finally, the coordinate-space representation techniques can also, in principle, be extended to include the state-dependent effects of realistic potentials. Work is in progress on each of these fronts.

\section{ACKNOWLEDGMENTS}

This work was developed under an "Acciones Integradas" research program between Spain and the United Kingdom. We gratefully acknowledge the financial support of the corresponding joint committee. Three of us (M.C.B., E.B., and R.G.) also acknowledge the support of the Comisión Interministerial de Ciencia y Tecnologia of Spain under Contract 969/87, while one of us (R.F.B.) acknowledges the support of a research grant from the Science and Engineering Research Council of Great Britain. 


\section{APPENDIX A: THE CC2 EQUATIONS}

In order to give a very brief discussion of the technical derivations of the actual equations solved and the necessary reduction of the matrix elements, we use the $\mathrm{CC} 2$ method as a typical example. The comparable derivations of the related terms in the other approximations are very similar. The starting point for the derivation is the basic CC2 wave function in the oscillator Fock space given by Eqs. (26) and (27). In terms of this CC2 wave function, the corresponding estimate for the energy is evaluated as usual by

$$
E=(4 !)^{-1 / 2}\left\langle 0\left|a_{000}^{4} H\right| \Psi_{2}\right\rangle \text {. }
$$

The cluster coefficients $\left\{S_{k}\right\}$ are similarly calculated by projecting the approximate CC2 Schrödinger equation onto the basis $\theta_{n}|0\rangle, n=1,2, \ldots$, to give the standard result

$$
\left\langle 0\left|\theta_{n}^{\dagger} H\right| \Psi_{2}\right\rangle=E\left\langle 0\left|\theta_{n}^{\dagger}\right| \Psi_{2}\right\rangle
$$

The Hamiltonian $H=T+V$ is expressed in secondquantized form in the harmonic-oscillator basis as usual,

$$
\begin{aligned}
& T=\sum_{i, j} T_{i j} a_{i}^{\dagger} a_{j}, \\
& V=\frac{1}{4} \sum_{i, j, k, l} V_{i j, k l} a_{i}^{\dagger} a_{j}^{\dagger} a_{l} a_{k},
\end{aligned}
$$

where we have used a single index $i$, for example, to represent the complete set of one-particle indices $\left\{n_{i} l_{i} m_{i}\right\}$. The matrix elements of the one-body kineticenergy operator may readily be reduced to the form

$$
T_{i j}=\delta_{l_{i} l_{j}} \delta_{m_{i} m_{j}} T\left(n_{i} l_{i} n_{j}\right)
$$

In the case of the Wigner-type (i.e., spin- and isospinindependent) forces with which we are solely concerned here, the potential matrix elements may be reduced to the form

$$
\begin{aligned}
V_{i j, k l}= & \sum_{\lambda, \mu}\left\langle l_{i} m_{i} l_{j} m_{j} \mid l_{i} l_{j} \lambda \mu\right\rangle\left\langle l_{k} m_{k} l_{l} m_{l} \mid l_{k} l_{l} \lambda \mu\right\rangle \\
& \times \sum_{n, n^{\prime}, N} \sum_{l, L}\left[1+(-1)^{l_{k}+l_{l}+L}\right]\left\langle n l N L \lambda \mid n_{i} l_{i} n_{j} l_{j} \lambda\right\rangle\left\langle n^{\prime} l N L \lambda \mid n_{k} l_{k} n_{l} l_{l} \lambda\right\rangle V\left(n l n^{\prime}\right)
\end{aligned}
$$

Our final forms for Eqs. (A1) and (A2) will thus now involve the reduced matrix elements $T\left(n l n^{\prime}\right)$ and $V\left(n l n^{\prime}\right)$, the Brody-Moshinsky and Clebsch-Gordan recoupling coefficients, and the cluster coefficients $\left\{S_{k}\right\}$ as unknowns. The further reduction of the equations to this final form is completely straightforward but tedious. It proceeds in two stages. In the first place, the vacuum expectation values of each of the products of creation and destruction operators must be evaluated using Wick's theorem. In fact, we did this by the use of algebraic computer routines. Secondly, a considerable amount of simplification occurs by use of the completeness relations for the various overlap integrals. A typical example which occurs in Eq. (A2) is

$$
\sum_{n, n^{\prime}} \sum_{n_{i} l_{l} m_{i}} \sum_{n_{j} l_{j} m_{j}}\left\langle l_{i} m_{i} l_{j} m_{j} \mid l_{i} l_{j} 00\right\rangle^{2}\left\langle n 0000 \mid n_{i} l_{i} n_{j} l_{j} 0\right\rangle\left\langle n^{\prime} 0000 \mid n_{i} l_{i} n_{j} l_{j} 0\right\rangle V\left(n 0 n^{\prime}\right) S_{n}=\sum_{n} V(n 0 n) S_{n}
$$

The final equations can be more compactly written in terms of the shorthand notations,

$$
\begin{aligned}
& \left\langle n \mid n_{i} l n_{j}\right\rangle_{0} \equiv\left\langle n 0000 \mid n_{i} \ln j l 0\right\rangle \\
& \mathcal{V}_{\lambda}\left(n_{1} l_{1} n_{2} l_{2} ; n_{3} l_{3} n_{4} l_{4}\right) \equiv \sum_{n n^{\prime} l} \sum_{N L}\left[1+(-1)^{l_{3}+l_{4}+L}\right]\left\langle n l N L \lambda \mid n_{1} l_{1} n_{2} l_{2} \lambda\right\rangle\left\langle n^{\prime} l N L \lambda \mid n_{3} l_{3} n_{4} l_{4} \lambda\right\rangle V\left(n l n^{\prime}\right)
\end{aligned}
$$

We also make liberal use of the particular value of a special case of Eq. (A7),

$$
\langle n \mid n 00\rangle_{0}=2^{-n} \text {. }
$$

By these means, Eq. (A1) for the CC2 estimate of the ground-state energy can be written as

$$
\begin{aligned}
E= & 4 T(000)+6 V(000)+6 \sum_{n}\left[2^{1-n} T(00 n)+\left(1+2^{2(1-n)}\right) V(00 n)\right] S_{n} \\
& +12 \sum_{n, n^{\prime}} 2^{-\left(n+n^{\prime}\right)}\left\langle n+n^{\prime} \mid n 0 n^{\prime}\right\rangle_{0} V\left(0,0, n+n^{\prime}\right) S_{n} S_{n^{\prime}} .
\end{aligned}
$$




$$
\begin{aligned}
& 0=2^{1-n} T(00 n)+\left(1+2^{2(1-n)}\right) V(00 n)+\left[\left(2+2^{2(1-n)}\right) T(000)+V(000)-\left(1+2^{2(1-n)}\right) E\right] S_{n} \\
& +2^{2-n} \sum_{n_{t}}\left\{2^{-n_{t}}\left[T\left(n 0 n_{i}\right)+\mathcal{V}_{0}\left(n 000 ; n_{i} 000\right)\right] S_{n_{t}}+\left\langle n+n_{i} \mid n_{i} 0 n\right\rangle_{0}\left[T\left(00 n_{i}\right)+2^{-n_{i}} V\left(00 n_{i}\right)\right] S_{n+n_{i}}\right\} \\
& +\sum_{n_{a} n_{b}} 2^{2-n_{b}}\left\langle n \mid n_{a} 0 n_{b}\right\rangle_{0}\left[T\left(00 n_{a}\right)+2^{-n_{a}} V\left(00 n_{a}\right)\right] S_{n_{b}} \\
& +2 \sum_{n_{a} n_{b} l}\left\langle n \mid n_{a} l n_{b}\right\rangle_{0} \sum_{n_{l}}\left\{2^{-n_{i}} \mathcal{V}_{0}\left(n_{a} l n_{b} l ; n_{i} 000\right) S_{n_{t}}+\left\langle n_{i}+n_{b}+l \mid n_{i} l n_{b}\right\rangle_{0}\left[T\left(n_{a} l n_{t}\right)+2 \mathcal{V}_{l}\left(n_{a} l 00 ; n_{i} l 00\right)\right] S_{n_{l}+n_{b}+l}\right\} \\
& +2^{1-n} \sum_{n_{a} n_{b} l}\left\langle n_{a}+n_{b}+l \mid n_{a} l n_{b}\right\rangle_{0} \mathcal{V}_{0}\left(n 000 ; n_{a} l n_{b} l\right) S_{n_{a}+n_{b}+l} \\
& +\frac{1}{2} \sum_{n_{a} n_{b} l} \sum_{n_{i} n_{j} l^{\prime}}\left\langle n \mid n_{a} l n_{b}\right\rangle_{0}\left\langle n_{i}+n_{j}+l^{\prime} \mid n_{i} l^{\prime} n_{j}\right\rangle_{0} \mathcal{V}_{0}\left(n_{a} l n_{b} l ; n_{i} l^{\prime} n_{j} l^{\prime}\right) S_{n_{i}+n_{j}+l^{\prime}} \\
& +\sum_{n_{a}}\left[2^{1-n_{a}} T\left(00 n_{a}\right)+V\left(00 n_{a}\right)\right] S_{n_{a}} S_{n}-E \sum_{n_{a} n_{b}} 2^{1-\left(n_{a}+n_{b}\right)}\left\langle n \mid n_{a} 0 n_{b}\right\rangle_{0} S_{n_{a}} S_{n_{b}} \\
& +4 \sum_{n_{a} n_{b} n_{l}} 2^{2-n_{b}}\left\langle n \mid n_{a} 0 n_{b}\right\rangle_{0}\left[\left\langle n_{a}+n_{i} \mid n_{i} 0 n_{a}\right\rangle_{0} T\left(00 n_{i}\right) S_{n_{b}} S_{n_{a}+n_{l}}+2^{-n_{i}} T\left(n_{a} 0 n_{i}\right) S_{n_{b}} S_{n_{t}}\right] \\
& +\sum_{n_{a} n_{b} n_{i} n_{j} l}\left\{\frac{2}{(2 l+1)}\left\langle n \mid n_{a} l n_{b}\right\rangle_{0}\left\langle n_{a}+n_{i}+l \mid n_{a} l n_{i}\right\rangle_{0}\left\langle n_{b}+n_{j}+l \mid n_{b} l n_{j}\right\rangle_{0}\right. \\
& \times\left\langle n_{i}+n_{j}+l \mid n_{i} l n_{j}\right\rangle_{0} V\left(0,0, n_{i}+n_{j}+l\right) S_{n+n_{i}-n_{b}} S_{n+n_{j}-n_{a}} \\
& +2^{1-n_{b}}\left\langle n \mid n_{a} 0 n_{b}\right\rangle_{0}\left\langle n_{i}+n_{j}+l \mid n_{i} \ln _{j}\right\rangle_{0} \mathcal{V}_{0}\left(n_{a} 000 ; n_{i} l n_{j} l\right) S_{n_{b}} S_{n_{i}+n_{j}+l} \\
& +2^{-n_{J}}\left\langle n \mid n_{a} l_{n_{b}}\right\rangle_{0}\left[2^{-n_{l}} \mathcal{V}_{0}\left(n_{a} l n_{b} l ; n_{i} 0 n_{j} 0\right) S_{n_{i}} S_{n_{j}}\right. \\
& \left.\left.+4\left\langle n_{i}+n_{b}+l \mid n_{i} l n_{b}\right\rangle_{0} \mathcal{V}_{l}\left(n_{a} l 00 ; n_{i} l n_{j} 0\right) S_{n_{j}} S_{n_{i}+n_{b}+l}\right]\right\} \text {. }
\end{aligned}
$$

(A11)

Equation (A11) holds for all $n=1,2, \ldots$, and it hence provides an infinite set of coupled nonlinear equations for the cluster coefficients $\left\{S_{n}\right\}$. As discussed in the text, this set of equations is truncated in practice by setting all coefficients $S_{l}=0$ for $l>n_{\max }$, and solving the lowest $n_{\max }$ equations exactly.

\section{APPENDIX B: RECURSION FORMULAS FOR GAUSSIAN MATRIX ELEMENTS}

When the interaction potential is a linear combination of Gaussian factors, as in the case of the $\mathbf{S} 3$ potential, it is possible to use recursive means to generate all of the integrals needed to compute the required matrix elements that arise in our various CI and CCM approximations. By expressing the associated Laguerre polynomials in their explicit form, ${ }^{71}$ the matrix elements of interest can all be written as a linear combination of the general quantities $D(p, q, n, m)$ defined as

$$
D(p, q, n, m) \equiv \int d^{3} r_{1} \cdots \int d^{3} r_{4} \exp \left[-\alpha^{2}\left(r_{1}^{2}+r_{2}^{2}+r_{3}^{2}+r_{4}^{2}\right)\right] \exp \left(-b r_{12}^{2}\right)\left(r_{i_{1} j_{1}}\right)^{2 p}\left(r_{i_{2} j_{2}}\right)^{2 q}\left(r_{i_{3} j_{3}}\right)^{2 n}\left(r_{i_{4} j_{4}}\right)^{2 m}
$$

where the first term in the integral corresponds to the square of the $(0 s)^{4}$ oscillator wave function, the second term is one of the Gaussian components of the potential with range $b^{-1 / 2}$, and the polynomial part (with integral values for the indices $p, q, n$, and $m$ ) may take several distinct topological forms according to the values of the indices $\left\{i_{n}, j_{n} ; n=1, \ldots, 4\right\}$, chosen from the set $\{1, \ldots, 4\}$. For ease, we maintain the interaction term fixed between the pair $(1,2)$ of particles. Furthermore, we may assume that all pairs $\left(i_{n}, j_{n}\right)$ are different, since we may obviously combine two like pairs into a single term. It is not difficult to show that the particular structure of the CCM wave function generates needed matrix elements that fall into four distinct categories. They generate the distinct topological forms

$$
\begin{aligned}
& \left(r_{12}\right)^{2(p+n)}\left(r_{34}\right)^{2(q+m)}, \\
& \left(r_{13}\right)^{2(p+n)}\left(r_{24}\right)^{2(q+m)}, \\
& r_{12}^{p} r_{13}^{n} r_{34}^{q} r_{24}^{m},
\end{aligned}
$$

and

$$
r_{13}^{p} r_{24}^{q} r_{14}^{n} r_{23}^{m}
$$


We first introduce, for each distinct topological form, a basic quantity $D$ defined as

$$
\begin{aligned}
D \equiv \int d^{3} r_{1} \cdots \int & d^{3} r_{4} \exp \left(-\alpha^{2} \sum_{l=1}^{4} r_{l}^{2}\right) \exp \left(-b r_{12}^{2}\right) \\
& \times \exp \left(\sum_{k=1}^{4} \lambda_{k} r_{i_{k} j_{k}}^{2}\right) .
\end{aligned}
$$

The general form of the integrals actually needed from Eq. (B1) may then be obtained by differentiation.

$D(p, q, n, m)=\left.\frac{\partial^{p}}{\partial \lambda_{1}^{p}} \frac{\partial^{q}}{\partial \lambda_{2}^{q}} \frac{\partial^{n}}{\partial \lambda_{3}^{n}} \frac{\partial^{m}}{\partial \lambda_{4}^{m}} D\right|_{\lambda_{1}=\cdots=\lambda_{4}=0}$.

Our main task is thus reduced to obtaining a simple method to handle Eq. (B3) after we have first computed $D$ from Eq. (B2). Clearly, this procedure has to be repeated for each distinct topology of the terms according to the choice of indices $\left\{i_{n}, j_{n}\right\}$.

The integral $D$ may be evaluated in Cartesian coordinates in terms of which it is the product of three fourdimensional integrals, one for each product of onedimensional integrals in the respective $x, y$, and $z$ variables. Each of these identical integrals has an integrand which is the exponential of a positive-definite quadratic form, namely, $\exp \left(-v_{i} E_{i j} v_{j}\right)$, where $v=x, y, z$, $i, j=1, \ldots, 4$, and the summation convention is implied. Thus, $D$ may be immediately evaluated as

$$
D=\pi^{6}(\operatorname{det} E)^{-3 / 2} .
$$

The $4 \times 4$ matrix $E$ depends on the oscillator parameter $\alpha$, the parameter $b$ which determines the range of the specific Gaussian term in the potential, and on the four subsidiary parameters $\lambda_{1}, \ldots, \lambda_{4}$. The explicit form of $E_{i j}$ depends upon the specific topological structure of the integrand. However, a very important property common to all forms is that $\operatorname{det} E$ is a multinomial in the parame- ters $\lambda_{i}$ which involves no higher than first powers in each parameter. It is not difficult to verify that the quadratic terms, which at first sight might be expected to appear, in fact vanish identically. More explicitly, we have the general form

$$
\operatorname{det} E=\sum_{i=0}^{1} \sum_{j=0}^{1} \sum_{k=0}^{1} \sum_{l=0}^{1} P_{i j k l} \lambda_{1}^{i} \lambda_{2}^{j} \lambda_{3}^{k} \lambda_{4}^{l}
$$

We observe from Eq. (B3) that we now require the partial derivatives of the multinomial in Eq. (B5) raised to the power $-\frac{3}{2}$. In order to establish a recurrence relation, it is now convenient to introduce a further quantity that slightly generalizes Eq. (B3), namely,

$$
D(p, q, n, m ; s) \equiv \frac{\partial^{p}}{\partial \lambda_{1}^{p}} \frac{\partial^{q}}{\partial \lambda_{2}^{q}} \frac{\partial^{n}}{\partial \lambda_{3}^{n}} \frac{\partial^{m}}{\partial \lambda_{4}^{m}}(\operatorname{det} E)^{-s} .
$$

We note, in particular, that, in comparison to Eq. (B3), the trivial factor $\pi^{6}$ has been removed, and the partial derivatives are no longer evaluated at the point $\lambda_{1}=\cdots=\lambda_{4}=0$. Thus, the factor $D(p, q, n, m ; s)$ is still a function of the parameters $\left\{\lambda_{k}\right\}$. The partial derivatives may now be performed recursively by making use of the simple relation

$$
\frac{d^{n}}{d x^{n}}[f(x)]^{-s}=-s f^{\prime}(x) \frac{d^{n-1}}{d x^{n-1}}[f(x)]^{-s-1},
$$

which is valid when $f^{\prime \prime}(x)=0$, for each of the four variables $\lambda_{1}, \ldots, \lambda_{4}$. In particular, use of Eq. (B7) with $x$ replaced by $\lambda_{1}$ readily yields the relation

$D(p, 0,0,0 ; s)=-s P_{1 j k l} \lambda_{2}^{j} \lambda_{3}^{k} \lambda_{4}^{l} D(p-1,0,0,0 ; s+1)$,

where here and henceforth we employ the summation convention over the indices $(i, j, k, l)$, each of which takes the values 0 or 1 only. Repeated partial differentiation of Eq. (B8) with respect to the remaining variables $\lambda_{2}, \lambda_{3}$, and $\lambda_{4}$ easily yields the final result,

$$
\begin{aligned}
D(p, q, n, m ; s)=-s & P_{1 j k l} \lambda_{2}^{j} \lambda_{3}^{k} \lambda_{4}^{l} D(p-1, q, n, m ; s+1)+q P_{11 k l} \lambda_{3}^{k} \lambda_{4}^{l} D(p-1, q-1, n, m ; s+1) \\
& +n P_{1 j 1 l} \lambda \lambda_{2}^{j} \lambda_{4}^{l} D(p-1, q, n-1, m ; s+1)+m P_{1 j k 1} \lambda_{2}^{j} \lambda_{3}^{k} D(p-1, q, n, m-1 ; s+1) \\
& +q n P_{111} \lambda_{4}^{l} D(p-1, q-1, n-1, m ; s+1)+q m P_{11 k 1} \lambda_{3}^{k} D(p-1, q-1, n, m-1 ; s+1) \\
& \left.+n m P_{1 j 11} \lambda{ }_{2}^{j} D(p-1, q, n-1, m-1 ; s+1)+q n m P_{1111} D(p-1, q-1, n-1, m-1 ; s+1)\right] .
\end{aligned}
$$

In practice, the recursion relation of Eq. (B9) is utilized by putting all $\lambda_{i}=0, i=1, \ldots, 4$, and using the initial value

$$
D(0,0,0,0 ; s)=(\operatorname{det} E)^{-s} .
$$

It is initialized by setting $s=\frac{3}{2}+4 N$, where $N$ is the maximum value of the indices $p, q, n$, and $m$ required.
We note that the normalization matrix elements between the uncorrelated oscillator states are also easily found as the particular case of Eq. (B1) where $b=0$. Similarly, the necessary matrix elements involving the kinetic-energy operator can also be cast into the general form of Eq. (B1) after a simple transformation of the operators $\nabla_{i}^{2}$. 
${ }^{1}$ H. Kümmel, Nucl. Phys. A176, 205 (1971).

${ }^{2}$ H. Kümmel and K. H. Lührmann, Nucl. Phys. A191, 525 (1972).

${ }^{3}$ K. H. Lührmann and H. Kümmel, Nucl. Phys. A194, 225 (1972).

4J. G. Zabolitzky, Nucl. Phys. A228, 272 (1974); A228, 285 (1974).

${ }^{5}$ R. Offermann, W. Ey, and H. Kümmel, Nucl. Phys. A273, 349 (1976); R. Offermann, ibid. A273, 368 (1976); W. Ey, ibid. A296, 189 (1978).

${ }^{6}$ H. Kümmel, K. H. Lührmann, and J. G. Zabolitzky, Phys. Rep. C 36, 1 (1978).

${ }^{7}$ J. G. Zabolitzky and W. Ey, Nucl. Phys. A328, 507 (1979).

8 J. G. Zabolitzky, Phys. Lett. 100B, 5 (1981).

${ }^{9}$ K. Emrich, Nucl. Phys. A351, 379 (1981); A351, 397 (1981); K. Emrich and J. G. Zabolitzky, ibid. A351, 439 (1981).

${ }^{10}$ H. G. Kümmel, in Nucleon-Nucleon Interaction and Nuclear Many-Body Problems, edited by S. S. Wu and T. T. S. Kuo (World-Scientific, Singapore, 1984), p. 46.

${ }^{11}$ J. Čižek, J. Chem. Phys. 45, 4256 (1966); Adv. Chem. Phys. 14, 35 (1969).

${ }^{12}$ I. Lindgren, Int. J. Quantum Chem. Symp. 12, 33 (1978).

${ }^{13}$ R. J. Bartlett and G. D. Purvis, Int. J. Quantum Chem. 14, 561 (1978); Phys. Scr. 21, 251 (1980).

${ }^{14}$ R. J. Bartlett, Annu. Rev. Phys. Chem. 32, 359 (1981).

${ }^{15}$ V. Kvasnička, V. Laurinc, and S. Biskupič, Phys. Rep. C 90, 160 (1982).

${ }^{16}$ K. Szalewicz, J. G. Zabolitzky, B. Jeziorski, and H. J. Monkhorst, J. Chem. Phys. 81, 2723 (1984).

${ }^{17}$ U. Kaldor, J. Chem. Phys. 87, 467 (1984).

${ }^{18}$ S. Pal, M. Rittby, R. J. Bartlett, D. Sinha, and D. Mukherjee, Chem. Phys. Lett. 137, 273 (1987).

${ }^{19}$ R. J. Bartlett, J. Phys. Chem. 93, 1697 (1989).

${ }^{20}$ R. F. Bishop and K. H. Lührmann, Phys. Rev. B 17, 3757 (1978); 26, 5523 (1982).

${ }^{21}$ J. Arponen and E. Pajanne, J. Phys. C 15, 2665 (1982); 15, 2683 (1982).

${ }^{22}$ K. Emrich and J. G. Zabolitzky, Phys. Rev. B 30, 2049 (1984).

${ }^{23}$ B. D. Day and J. G. Zabolitzky, Nucl. Phys. A366, 221 (1981).

${ }^{24}$ P. Bodden, Nucl. Phys. A384, 449 (1982).

${ }^{25}$ C. S. Hsue and J. L. Chern, Phys. Rev. D 29, 643 (1984).

${ }^{26}$ U. B. Kaulfuss and M. Altenbokum, Phys. Rev. D 33, 3658 (1986).

${ }^{27}$ H. G. Kümmel, in Condensed Matter Theories, edited by J. S. Arponen, R. F. Bishop, and M. Manninen (Plenum, New York, 1988), Vol. 3, p.21.

${ }^{28}$ R. F. Bishop and M. F. Flynn, Phys. Rev. A 38, 2211 (1988).

${ }^{29}$ R. F. Bishop, M. C. Boscá, and M. F. Flynn, Phys. Lett. A 132, 440 (1988); Phys. Rev. A 40, 3484 (1989).

${ }^{30}$ H. Kümmel, Phys. Rev. C 27, 765 (1983).

${ }^{31}$ G. Hasberg and H. Kümmel, Phys. Rev. C 33, 1367 (1986).

${ }^{32}$ U. Kaulfuss, Phys. Rev. D 32, 1421 (1985).

${ }^{33}$ C. S. Hsue, H. Kümmel, and P. Ueberholz, Phys. Rev. D 32, 1435 (1985).

${ }^{34}$ M. Altenbokum and H. Kümmel, Phys. Rev. D 32, 2014 (1985).

${ }^{35}$ U. Kaulfuss and M. Altenbokum, Phys. Rev. D 35, 609 (1987).

${ }^{36}$ M. Funke, U. Kaulfuss, and H. Kümmel, Phys. Rev. D 35, 621 (1987).

${ }^{37}$ R. F. Bishop and H. G. Kümmel, Phys. Today 40(3), 52 (1987).

${ }^{38}$ R. F. Bishop, in Nucleon-Nucleon Interaction and Nuclear Many-Body Problems, edited by S. S. Wu and T. T. S. Kuo (World Scientific, Singapore, 1984), p. 604.
${ }^{39}$ R. F. Bishop, An. Fis. A 81, 9 (1985).

${ }^{40}$ R. F. Bishop, W. Piechocki, and G. A. Stevens, Few-Body Syst. 4, 161 (1988); 4, 179 (1988).

${ }^{41}$ M. Altenbokum, K. Emrich, H. Kümmel, and J. G. Zabolitzky, in Condensed Matter Theories, edited by P. Vashishta, R. K. Kalia, and R. F. Bishop (Plenum, New York, 1987), Vol. 2, p. 389.

42J. Arponen, Ann. Phys. (NY) 151, 311 (1983).

${ }^{43}$ J. S. Arponen, R. F. Bishop, and E. Pajanne, Phys. Rev. A 36, 2519 (1987); 36, 2539 (1987).

${ }^{44}$ J. S. Arponen, R. F. Bishop, E. Pajanne, and N. I. Robinson, Phys. Rev. A 37, 1065 (1988).

45J. Arponen, R. F. Bishop, and E. Pajanne, in Condensed Matter Theories, edited by P. Vashishta, R. K. Kalia, and R. F. Bishop (Plenum, New York, 1987), Vol. 2, p. 373.

${ }^{46}$ N. I. Robinson, R. F. Bishop, and J. Arponen, Phys. Rev. A 40, 4256 (1989).

${ }^{47}$ H. J. Lipkin, N. Meshkov, and A. J. Glick, Nucl. Phys. 62, 188 (1965); 62, 199 (1965); 62, 211 (1965).

${ }^{48}$ F. Coester, Nucl. Phys. 7, 421 (1958); F. Coester and H. Kümmel, ibid. 17, 477 (1960).

${ }^{49}$ I. R. Afnan and Y. C. Tang, Phys. Rev. 175, 1337 (1968).

${ }^{50}$ R. A. Malfliet and J. A. Tjon, Nucl. Phys. A127, 161 (1969).

${ }^{51}$ R. F. Bishop, M. F. Flynn, M. C. Boscá, E. Buendía, and R. Guardiola, in The Nuclear Equation of State (Part A: Discovery of Nuclear Shock Waves and EOS), edited by W. Greiner (Plenum, New York, 1990), p. 605.

52 J. J. Bevalacqua and R. J. Philpott, Nucl. Phys. A275, 301 (1977).

${ }^{53}$ J. J. Bevalacqua, Can. J. Phys. 57, 1833 (1979); Phys. Rev. C 28, 2540 (1983).

${ }^{54}$ R. Ceuleneer and P. Vandepeutte, Phys. Rev. C 31, 1528 (1985).

${ }^{55}$ R. Ceuleneer, P. Vandepeutte, and C. Semay, Phys. Lett. B 196, 303 (1987).

${ }^{56}$ R. F. Bishop, M. F. Flynn, and M. Znojil, Phys. Rev. A 39, 5336 (1989).

${ }^{57}$ H. Feshbach, Ann. Phys. (NY) 5, 357 (1958); P. O. Löwdin, in Perturbation Theory and its Applications in Quantum Mechanics, edited by C. H. Wilcox (Wiley, New York, 1966), p. 255.

${ }^{58}$ C. Bloch, Nucl. Phys. 6, 329 (1958); 7, 451 (1958).

${ }^{59}$ I. Lindgren, J. Phys. B 7, 2441 (1974).

${ }^{60}$ S. Salomonson, I. Lindgren, and A. M. Martensson, Phys. Scr. 21, 351 (1980); I. Lindgren and J. Morrison, Atomic ManyBody Theory, 2nd ed. (Springer, Berlin, 1986).

${ }^{61}$ R. K. Nesbet, Phys. Rev. 109, 1632 (1958).

${ }^{62} \mathrm{H}$. Primas, in Modern Quantum Chemistry, edited by $\mathrm{O}$. Sinanoglu (Academic, New York, 1965), Vol. II, p. 45.

${ }^{63}$ K. A. Brueckner, Phys. Rev. 97, 1353 (1955); 100, 36 (1955).

${ }^{64}$ J. Goldstone, Proc. R. Soc. London Ser. A 239, 267 (1957).

${ }^{65}$ N. M. Hugenholtz, Physica 23, 481 (1957).

66J. Hubbard, Proc. R. Soc. London Ser. A 240, 539 (1957).

${ }^{67}$ B. Brandow, Rev. Mod. Phys. 39, 771 (1967).

${ }^{68}$ D. Mukherjee, R. K. Moitra, and A. Mukhopadhyay, Mol. Phys. 30, 1861 (1975); 33, 955 (1977).

${ }^{69}$ D. Mukherjee, Chem. Phys. Lett. 125, 207 (1986); Int. J. Quantum Chem. Symp. 20, 409 (1986).

${ }^{70}$ I. Lindgren and D. Mukherjee, Phys. Rep. 151, 93 (1987); R. Chowdhuri, D. Mukherjee, and M. D. Prasad, in Aspects of Many-Body Effects in Molecules and Extended Systems, Vol. 50 of Lecture Notes in Chemistry, edited by D. Mukherjee (Springer, Berlin, 1989), p. 3.

${ }^{71}$ A. Erdélyi et al., Higher Transcendental Functions (McGraw- 
Hill, New York, 1954), Vol. II, p. 188.

${ }^{72}$ D. J. Thouless, The Quantum Mechanics of Many Body Systems (Academic, New York, 1961); Nucl. Phys. 21, 225 (1960).

${ }^{73}$ T. A. Brody and M. Moshinsky, Tables of Transformation Brackets for Nuclear Shell-Model Calculations, 2nd ed. (Gordon and Breach, New York, 1967).

${ }^{74}$ J. B. McGrory and B. H. Wildenthal, Phys. Lett. 60B, 5 (1975).

${ }^{75}$ R. M. Mendez-Moreno and T. H. Seligman, Ann. Phys. (NY) 88, 397 (1974).

${ }^{76}$ D. E. Knuth, The Art of Computer Programming (AddisonWesley, Reading, Mass., 1973), Vol. III, Sec. 6.4.

${ }^{77}$ M. Fink, Nucl. Phys. A221, 163 (1974).
${ }^{78}$ J. G. Zabolitzky and M. H. Kalos, Nucl. Phys. A356, 114 (1981); J. G. Zabolitzky, K. E. Schmidt, and M. H. Kalos, Phys. Rev. C 25, 1111 (1982).

${ }^{79}$ L. Majling, J. Řízek, and Z. Pluhař, Czech, J. Phys. 24B, 1306 (1974); L. Majling, J. Řizek, Z. Pluhař, and Y. F. Smirnov, J. Phys. G 2, 357 (1976).

${ }^{80}$ L. Bracci, S. Rosati, and M. Viviani, in Proceedings of the Secondo Convegno su Problemi di Física Nucleare Teorica, edited by L. Bracci et al. (ETS editrice, Pisa, 1988), p. 34.

${ }^{81}$ M. Fabre de la Ripelle, H. Fiedeldey, and A. Sofianos, Phys. Rev. C 38, 449 (1988).

${ }^{82}$ M. Fabre de la Ripelle, C. R. Acad. Sci. Paris 306, 1313 (1988). 